\title{
The $(1-\mathbb{E})$-transform in combinatorial Hopf algebras
}

\author{
Florent Hivert • Jean-Gabriel Luque • \\ Jean-Christophe Novelli · Jean-Yves Thibon
}

Received: 2 April 2010 / Accepted: 28 June 2010 / Published online: 20 July 2010

(C) Springer Science+Business Media, LLC 2010

\begin{abstract}
We extend to several combinatorial Hopf algebras the endomorphism of symmetric functions sending the first power-sum to zero and leaving the other ones invariant. As a "transformation of alphabets", this is the $(1-\mathbb{E})$-transform, where $\mathbb{E}$ is the "exponential alphabet," whose elementary symmetric functions are $e_{n}=\frac{1}{n !}$. In the case of noncommutative symmetric functions, we recover Schocker's idempotents for derangement numbers (Schocker, Discrete Math. 269:239-248, 2003). From these idempotents, we construct subalgebras of the descent algebras analogous to the peak algebras and study their representation theory. The case of WQSym leads to similar subalgebras of the Solomon-Tits algebras. In FQSym, the study of the transformation boils down to a simple solution of the Tsetlin library in the uniform case.
\end{abstract}

Keywords Combinatorial Hopf algebras · Symmetric functions · Derangements

\footnotetext{
F. Hivert · J.-G. Luque

LITIS, Université de Rouen, Avenue de l'université, 76801 Saint Étienne du Rouvray, France

F. Hivert

e-mail: hivert@univ-rouen.fr

J.-G. Luque

e-mail: luque@univ-rouen.fr

J.-C. Novelli · J.-Y. Thibon ( $₫)$

Institut Gaspard Monge, Université de Marne-la-Vallée, 5, Boulevard Descartes, Champs-sur-Marne, 77454 Marne-la-Vallée cedex 2, France

e-mail: jyt@univ-mlv.fr

J.-C. Novelli

e-mail: novelli@univ-mlv.fr
} 


\section{Virtual alphabets in combinatorial Hopf algebras}

The notion of virtual alphabet provides a powerful symbolic notation for dealing with endomorphisms of combinatorial Hopf algebras, at least for the combinatorial Hopf algebras which can be realized in terms of (noncommutative) polynomials. Examples include Sym (noncommutative symmetric functions, [12, 19]), FQSym (Free quasi-symmetric functions [9, 10], which realize the Malvenuto-Reutenauer algebra [23]), WQSym (Word quasi-symmetric functions [18]), PQSym (Parking quasisymmetric functions [28]), and their subalgebras as long as they are stable under the internal product. These algebras can be regarded as generalizations of the Hopf algebra of symmetric functions, for which this formalism was essentially promoted by Lascoux [20, 21].

The algebra of symmetric functions is denoted by Sym. Apart from this detail, our notation for symmetric functions follows Macdonald's book [22].

\subsection{Virtual alphabets for symmetric functions}

It is convenient to use the generic term alphabet to designate the argument of a symmetric function. Indeed, a symmetric function $f$ is characterized by its expression $f\left(x_{1}, x_{2}, \ldots\right)$ in terms of monomials in an infinite sequence of independent indeterminates $x_{i}$, to which various sets or multisets of algebraic expressions (numbers, monomials, cohomology classes, vector bundles, etc.) can be substituted. The diversity of possible interpretations suggests to treat as far as possible these arguments as formal symbols (or letters, whence the term alphabet), and the possible occurrence of multiple arguments suggests to extend the usual meaning of this term and to understand it as a multiset of symbols.

Actually, a multiset $A=\{a, c, c, c, f, f\}$ is nothing but a formal linear combination of symbols with nonnegative integer coefficients, and it is more convenient to represent it as $A=a+3 c+2 f$. With this notation, the union of multisets becomes just a sum $A+B$, and if we have sums, we can also have differences $A-B$, at least when $B$ is contained in $A$.

This leads us to the point of this paragraph. When $B$ is not contained in $A$, let us call the formal combination $A-B$ a virtual alphabet. It is easy to define the value of a symmetric function on a virtual alphabet. Indeed, it is well known that any symmetric function $f$ can be expressed in terms of the elementary symmetric functions $e_{k}$. The elementary functions $e_{k}(A)$ of a genuine alphabet $A$ are the coefficients of the product

$$
\lambda_{t}(A):=\prod_{a \in A}(1+t a)=\sum_{k} e_{k}(A) t^{k}
$$

and when $B$ is contained in $A$, those of the alphabet $C=A-B$ are obtained by division of the generating functions:

$$
\lambda_{t}(A-B)=\lambda_{t}(A) / \lambda_{t}(B) .
$$

When $B$ is not contained in $A$, this still defines coefficients $e_{k}(A-B)$, which are, by definition, the elementary symmetric functions of the virtual alphabet $A-B$. 
This defines all symmetric functions of $A-B$, and one has, for example, the simple expression $p_{n}(A-B)=p_{n}(A)-p_{n}(B)$ for the power sums.

More generally, a virtual alphabet can be defined by any sequence $\left(e_{n}(A)\right)_{n \geq 1}$, interpreted as its elementary symmetric functions (or as any sequence of independent generators of the algebra of symmetric functions, such as power sums $p_{n}$ or complete homogeneous functions $h_{n}$ ).

For example, although the exponential function $e^{t}$ has no zeros in the complex plane, we can introduce a virtual alphabet $\mathbb{E}$ such that

$$
e_{n}(\mathbb{E})=\frac{1}{n !}, \quad \lambda_{t}(\mathbb{E})=e^{t}
$$

This is a useful trick, allowing to understand a lot of formulas in combinatorics or analysis as specializations of simple identities on symmetric functions. For example, the exponential generating function of the derangement numbers $d_{n}$ is just

$$
\sigma_{t}(1-\mathbb{E}):=\lambda_{-t}(1-\mathbb{E})^{-1}=\frac{e^{-t}}{1-t} .
$$

Also, for a partition $\lambda$ of $n, n ! s_{\lambda}(\mathbb{E})=f_{\lambda}$, the number of standard tableaux of shape $\lambda$ (the $s_{\lambda}$ are the Schur functions). Another virtual alphabet of interest is $1-q$, where $p_{n}(1-q)=1-q^{n}$. It plays an essential role in the theory of Hall-Littlewood functions [22].

Our expression of alphabets as formal sums or differences also allows the consideration of products. For genuine alphabets $A, B$,

$$
A B=\{a b \mid a \in A, b \in B\}=\sum_{a} a \sum_{b} b
$$

and for virtual alphabets, the symmetric functions of $A B$ are defined by any of the formulas like $p_{n}(A B)=p_{n}(A) p_{n}(B)$ or

$$
h_{n}(A B)=\sum_{\lambda \vdash n} s_{\lambda}(A) s_{\lambda}(B)
$$

the famous Cauchy identity More generally, for any function $f$, we have: $f(A X)=$ $f(X) * \sigma_{1}(A X)$, where $*$ denotes the internal product, and $\sigma_{t}(X)=\sum t^{n} h_{n}(X)$ is the generating series of homogeneous complete functions.

Hence, with this formalism, we can consider transformations of alphabets on symmetric functions, which are ring homomorphisms mapping any $f(X)$ to $f(\mathbb{E} X)$, $f((1-\mathbb{E}) X), f((1-q) X), f(X /(1-q))$, and so on. Remark that in this setting, the virtual alphabet $1-q$ is the inverse of the genuine alphabet $\left\{1, q, q^{2}, \ldots\right\} \equiv$ $1+q+q^{2}+\cdots$.

Actually, Sym is a $\lambda$-ring $[20,21]$, and the notion of plethysm allows much more complicated transformations, but in the present paper, we concentrate on the above mentioned ones since these can be defined only in terms of the (combinatorial) Hopf algebra structure. 


\subsection{Extension to combinatorial Hopf algebras}

Let $X$ and $Y$ be two infinite alphabets. Identifying expressions like $f(X) g(Y)$ with the tensor product $f \otimes g$, we can regard the transformations

$$
\Delta: f(X) \longmapsto f(X+Y)
$$

and

$$
\delta: f(X) \longmapsto f(X Y)
$$

as linear maps $S y m \rightarrow S y m \otimes S y m$, that is, as comultiplications. Moreover, both are (obviously) algebra morphisms, and the first one being graded in the sense that

$$
\Delta: \operatorname{Sym}_{n} \longrightarrow \bigoplus_{i+j=n} \operatorname{Sym}_{i} \otimes \operatorname{Sym}_{j}
$$

defines actually a Hopf algebra structure, whose antipode is simply $f(X) \mapsto f(-X)$.

Clearly, the transformations $f(\mathbb{E} X), f((1-\mathbb{E}) X), f((1-q) X$ and so on can be defined only in terms of these coproducts of the antipode, and of the internal product. It turns out that most combinatorial Hopf algebras can be realized in terms of polynomials in some infinite and totally ordered alphabet, denoted by $A=\left\{a_{n} \mid n \geq 1\right\}$ in the case of noncommuting letters, and by $X=\left\{x_{n} \mid n \geq 1\right\}$ in the case of commuting letters. The basic example is the pair Noncommutative Symmetric Functions-Quasisymmetric functions (Sym, QSym) of mutually dual combinatorial Hopf algebras. Sums and differences of alphabets are defined on both sides, and it is possible to make sense of the product $X A$ (see [19]). Hence, our transformations are defined in this case.

The subject of this article is the study of the $(1-\mathbb{E})$-transform in the pair (Sym, QSym), and its extension to other combinatorial Hopf algebras.

\section{The $(1-\mathbb{E})$-transform in Sym}

\subsection{Background}

Our notations for the Hopf algebra of noncommutative symmetric functions are as in $[12,19]$. This Hopf algebra is denoted by $\operatorname{Sym}$, or by $\operatorname{Sym}(A)$ if we consider the realization in terms of an auxiliary alphabet. Bases of its homogeneous component $\mathbf{S y m}_{n}$ are labelled by compositions $I=\left(i_{1}, \ldots, i_{r}\right)$ of $n$. The noncommutative complete and elementary functions are denoted by $S_{n}$ and $\Lambda_{n}$, and the notation $S^{I}$ means $S_{i_{1}} \cdots S_{i_{r}}$. The ribbon basis is denoted by $R_{I}$. The notation $I \vDash n$ means that $I$ is a composition of $n$. The conjugate composition is denoted by $I^{\sim}$, the mirror image composition by $\bar{I}$. The descent set of $I$ is $\operatorname{Des}(I)=\left\{i_{1}, i_{1}+i_{2}, \ldots, i_{1}+\cdots+i_{r-1}\right\}$.

The graded dual of Sym is $Q S y m$ (quasi-symmetric functions). The dual basis of $\left(S^{I}\right)$ is $\left(M_{I}\right)$ (monomial), and that of $\left(R_{I}\right)$ is $\left(F_{I}\right)$.

The Hopf structures on Sym and QSym allows one to partially extend the $\lambda$-ring notation of ordinary symmetric functions (see [19], and [20] for background on 
the original commutative version). If $A$ and $X$ represent totally ordered sets of noncommuting and commuting variables, respectively, the noncommutative symmetric functions of $X A$ are defined by

$$
\sigma_{t}(X A)=\sum_{n \geq 0} t^{n} S_{n}(X A)=\prod_{x \in X}^{\rightarrow} \sigma_{t x}(A)=\sum_{I} t^{|I|} M_{I}(X) S^{I}(A) .
$$

Now, $X$ can be a virtual alphabet, defined by an arbitrary specialization of an independent set of generators of $Q S y m$. An alternative way to express the transformation of alphabets defined by $X$ is [19]

$$
F(X A)=F(A) * \sigma_{1}(X A)
$$

where $*$ is the internal product. Since $\sigma_{1}(X A)$ is grouplike, the $X$-transform is a bialgebra morphism, thanks to the splitting formula

$$
\left(F_{1} F_{2} \cdots F_{r}\right) * G=\mu_{r}\left[\left(F_{1} \otimes \cdots \otimes F_{r}\right) * \Delta^{r} G\right]
$$

where in the right-hand side, $\mu_{r}$ denotes the $r$-fold ordinary multiplication and $*$ stands for the operation induced on $\mathbf{S y m}^{\otimes n}$ by $*$.

Thanks to the commutative image homomorphism Sym $\rightarrow S y m$, noncommutative symmetric functions can be evaluated on any element $x$ of a $\lambda$-ring, $S_{n}(x)$ being $S^{n}(x)$, the $n$th symmetric power. Recall that $x$ is said of rank one (resp. binomial) if $\sigma_{t}(x)=(1-t x)^{-1}\left(\right.$ resp. $\left.\sigma_{t}(x)=(1-t)^{-x}\right)$. The scalar $x=1$ is the only element having both properties. We usually consider that our auxiliary variable $t$ is of rank one, so that $\sigma_{t}(A)=\sigma_{1}(t A)$.

The argument $A$ of a noncommutative symmetric function can be a virtual alphabet. This means that, being algebraically independent, the $S_{n}$ can be specialized to any sequence $\alpha_{n} \in \mathcal{A}$ of elements of any associative algebra $\mathcal{A}$. Writing $\alpha_{n}=S_{n}(A)$ defines all the symmetric functions of $A$. Quasi-symmetric functions of a virtual alphabet $X$ can be defined by a specialization of the algebraic generators of $Q S y m$, which is more easily done by expressing the noncommutative symmetric functions of $X A$ in terms of $A$.

The specializations $X=\mathbb{E}$, defined by

$$
S_{n}(\mathbb{E} A)=\frac{1}{n !} S_{1}(A)^{n}
$$

(so that $\left.F_{I}(\mathbb{E})=\frac{1}{n !}\right)$ and $X=\frac{1}{1-q}$, for which

$$
S_{n}\left(\frac{A}{1-q}\right)=\frac{1}{(q)_{n}} \sum_{I \models n} q^{\operatorname{maj}(I)} R_{I}(A)
$$

are of special importance. The second one can be used to define the peak algebras and simplifies considerably their investigation $[1,3,24]$. Here, we will study the $(1-\mathbb{E})$ transform by the methods developed in these references. 


\subsection{Noncommutative derangement numbers}

A possible motivation for the $(1-\mathbb{E})$-transforms is the combinatorics of derangements. Indeed, as already mentioned, the generating function of the derangement numbers

$$
D(t)=\sum_{n \geq 0} d_{n} \frac{t^{n}}{n !}=\frac{e^{-t}}{1-t}
$$

can be expressed as

$$
D(t)=\sigma_{1}((1-\mathbb{E}) t) .
$$

The specializations of the various bases of symmetric functions at $1-\mathbb{E}$ are obtained by expanding the Cauchy kernel $\sigma_{1}((1-\mathbb{E}) X)$, and analogously, its quasi-symmetric functions can be defined as the coefficients of the expansion of the noncommutative Cauchy kernel

$$
\sigma_{1}((1-\mathbb{E}) A)=e^{-S_{1}(A)} \sigma_{1}(A)
$$

on any basis of noncommutative symmetric functions.

For example, since the quasi-monomial basis $M_{I}$ is dual to $S^{I}$, we have

$$
\sigma_{1}((1-\mathbb{E}) A)=\sum_{n \geq 0} S_{n}((1-\mathbb{E}) A)=\sum_{I} M_{I}(1-\mathbb{E}) S^{I}(A),
$$

so that

$$
\begin{aligned}
& S_{1}((1-\mathbb{E}) A)=0, \quad S_{2}((1-\mathbb{E}) A)=S_{2}(A)-S^{11}(A) / 2, \\
& S_{3}((1-\mathbb{E}) A)=S_{3}(A)-S^{12}(A)+S^{111}(A) / 3,
\end{aligned}
$$

hence, implying

$$
M_{1}(1-\mathbb{E})=0, \quad M_{2}(1-\mathbb{E})=1, \quad M_{11}(1-\mathbb{E})=-1 / 2,
$$

and

$$
\begin{array}{ll}
M_{3}(1-\mathbb{E})=1, & M_{21}(1-\mathbb{E})=-1, \\
M_{12}(1-\mathbb{E})=0, & M_{111}(1-\mathbb{E})=1 / 3 .
\end{array}
$$

Since for $A=1, S_{n}((1-\mathbb{E}) A)=d_{n} / n$ !, these noncommutative symmetric functions might be called noncommutative derangement numbers (see [29] for other examples of noncommutative combinatorial numbers).

Another natural noncommutative analogue of $d_{n}$ is given by

$$
\lambda_{-t}(A)\left(1-t S_{1}(A)\right)^{-1}
$$


which gives back the $d_{n}$ for $A=\mathbb{E}$, and $d_{n}(q)$ for $A=\frac{1}{1-q}$. Its expansion of the ribbon basis is easily seen to be

$$
\sum_{n \geq 0} t^{n} \sum_{i=1}^{[n / 2]} \sum_{|J|=n-2 i} R_{12 i_{\triangleright} J}
$$

(ribbons whose first column is of even height, or permutations whose position of the first local minimum is even; the observation that these permutations are counted by $d_{n}$ is due to Désarménien [5], and an explicit bijection has been given by Désarménien and Wachs $[6,7])$.

Similarly,

$$
\lambda_{u-t}(A)\left(1-t S_{1}(A)\right)^{-1}
$$

gives a natural noncommutative analog of the generating series $D(t, u)=e^{u-t} /$ $(1-t)$ for permutations by number of fixed points, and the expansion in FQSym of

$$
\sigma_{t}(A)\left(1-t S_{1}(A)\right)^{-1}
$$

yields a class of permutations in bijection with arrangements: for $A=\mathbb{E}$, this series is $\frac{e^{t}}{1-t}$.

Denote for short by a $\sharp$ the $(1-\mathbb{E})$ transform, i.e.,

$$
F^{\sharp}:=F((1-\mathbb{E}) A)=F * \sigma_{1}^{\sharp}
$$

where

$$
\sigma_{1}^{\sharp}=\sigma_{1}((1-\mathbb{E}) A)=e^{-S_{1}} \sigma_{1},
$$

that is,

$$
S_{n}^{\sharp}=\sum_{i=0}^{n}(-1)^{i} \frac{S_{1}^{i}}{i !} S_{n-i} .
$$

Lemma 2.1 The $\sharp$-transform is a projector, i.e.,

$$
\sigma_{1}^{\sharp} * \sigma_{1}^{\sharp}=\sigma_{1}^{\sharp},
$$

or equivalently,

$$
S_{n}^{\sharp} * S_{n}^{\sharp}=S_{n}^{\sharp} \quad \text { for all } n \text {. }
$$

Proof Since the $\sharp$ transform is a homomorphism,

$$
\begin{aligned}
\sigma_{1}^{\sharp} * \sigma_{1}^{\sharp} & =\left(e^{-S_{1}} \sigma_{1}\right) * \sigma_{1}^{\sharp}=\left(e^{-S_{1}} * \sigma_{1}^{\sharp}\right)\left(\sigma_{1} * \sigma_{1}^{\sharp}\right) \\
& =e^{-S_{1}^{\sharp}} \sigma_{1}^{\sharp}=\sigma_{1}^{\sharp} .
\end{aligned}
$$


Hence, $S_{n}^{\sharp}$ corresponds to an idempotent $\delta_{n}$ of the descent algebra $\Sigma_{n}$. We shall see later that the dimension of the representation $\mathbb{C S}_{n} \delta_{n}$ is the derangement number $d_{n}$ and that $\delta_{n}$ coincides with Schocker's derangement idempotent [32], whence the name derangement algebra below for the corresponding ideal of the descent algebra.

2.3 The small derangement algebra $\mathcal{D}^{(0)}$

\subsubsection{Definition of $\mathcal{D}^{(0)}$}

Imitating the construction of the peak algebra in [3], we define the small derangement algebra, or the derangement ideal as

$$
\mathcal{D}^{(0)}:=\mathbf{S y m}^{\sharp}=\mathbf{S y m}(A) * \sigma_{1}^{\sharp} .
$$

By definition of the transformation of alphabet, $\mathcal{D}^{(0)}$ is a Hopf subalgebra of Sym, and thanks to the second equality, each homogenous component $\mathcal{D}_{n}^{(0)}$ is a left ideal of $\mathbf{S y m}_{n}$ for the internal product, explaining the two names for $\mathcal{D}^{(0)}$, depending on which property we want to emphasize.

\subsubsection{Dimension and bases of $\mathcal{D}_{n}^{(0)}$}

We have $S_{1}^{\sharp}=0$, and the other $S_{n}^{\sharp}$ are clearly algebraically independent. Hence, the dimension $d_{n}^{(0)}$ of $\mathcal{D}_{n}^{(0)}$ is given by the number of compositions of $n$ with no part equal to 1 . These elements satisfy the induction

$$
d_{0}^{(0)}=1, \quad d_{1}^{(0)}=0, \quad d_{n}^{(0)}=d_{n-1}^{(0)}+d_{n-2}^{(0)} \quad \text { for } n \geq 2,
$$

hence are shifted Fibonacci numbers.

Let us say that a composition is nonunitary if it does not contain the part 1 . Then the $S^{I \sharp}$ with $I$ nonunitary form a basis of $\mathcal{D}_{n}^{(0)}$.

Since the coproducts

$$
\Delta S_{n}^{\sharp}=\sum_{i+j=n} S_{i}^{\sharp} \otimes S_{j}^{\sharp}
$$

are given by the same formula as those of the $S_{n}$, we see that $\mathcal{D}_{n}^{(0)}$ is isomorphic to the quotient $\mathbf{S y m}$ ' of Sym by the two-sided ideal generated by $S_{1}$. Hence, its (graded) dual is isomorphic to the subalgebra $Q S y m^{\prime}$ of $Q S y m$ spanned by the $M_{I}$ for $I$ nonunitary.

We shall see below (Proposition 2.2) that $\sigma_{1}^{\sharp}$ is neutran in $\mathcal{D}_{n}^{(0)}$. Since $\sigma_{1}^{\sharp}$ is a projector, we have

$$
f^{\sharp} * g^{\sharp}=(f * g)^{\sharp} .
$$

$\mathbf{S y m}^{\prime}$ being stable under the internal product, $\mathcal{D}_{n}^{(0)}$ is also $*$-isomorphic to $\mathbf{S y m}_{n}^{\prime}$.

Recall that the ribbon basis of Sym is given by

$$
R_{I}=\sum_{J \leq I} S^{J}
$$


where $\leq$ is the reverse refinement order. If $I$ is nonunitary, so are all $J \leq I$, thus

$$
Q_{I}:=R_{I}^{\sharp} \quad(1 \notin I)
$$

is a basis of $\mathcal{D}_{n}^{(0)}$.

\subsection{Algebraic structure of $\left(\mathcal{D}_{n}^{(0)}, *\right)$}

Since the construction of $\mathcal{D}^{(0)}$ mimics the one of the peak ideal, obtained in [3] as the image of the $(1-q)$-transform at $q=-1$, one may expect that $\mathcal{D}^{(0)}$ shares many properties with the peak ideal. There are some differences, however. Whilst the peak ideal has no unity for the internal product $*$, we have the following proposition.

Proposition 2.2 For all $n,\left(\mathcal{D}_{n}^{(0)}\right.$, *) is a unital algebra with $S_{n}^{\sharp}$ as neutral element.

Proof From Lemma 2.1, we already know that $\sigma_{1}^{\sharp}$ is neutral on the right. To prove that it is neutral on the left, let us consider its action on the generating series of a basis of $\mathcal{D}^{(0)}$

$$
\sigma_{1}(X \cdot(1-\mathbb{E}) A)=\sum_{I} M_{I}(X) S^{I \sharp}(A) .
$$

We have

$$
\sigma_{1}^{\sharp} * \sigma_{1}(X \cdot(1-\mathbb{E}) A)=\sigma_{1}^{\sharp} * \sigma_{1}(X A) * \sigma_{1}^{\sharp}
$$

and

$$
\begin{aligned}
\sigma_{1}^{\sharp} * \sigma_{1}(X A) & =\left(e^{-S_{1}(A)} \sigma_{1}(A)\right) * \sigma_{1}(X A) \\
& =\mu\left[\left(e^{-S_{1}(A)} \otimes \sigma_{1}(A)\right) * 2\left(\sigma_{1}(X A) \otimes \sigma_{1}(X A)\right)\right] \\
& =\left(e^{-S_{1}(A)} * \sigma_{1}(X A)\right)\left(\sigma_{1}(A) * \sigma_{1}(X A)\right) \\
& =e^{-S_{1}(X A)} \sigma_{1}(X A)
\end{aligned}
$$

so that

$$
\begin{aligned}
\sigma_{1}^{\sharp} * \sigma_{1}(X \cdot(1-\mathbb{E}) A) & =\left[e^{-S_{1}(X A)} \sigma_{1}(X A)\right] * \sigma_{1}^{\sharp} \\
& =e^{-S_{1}(X \cdot(1-\mathbb{E}) A)} \sigma_{1}(X \cdot(1-\mathbb{E}) A) \\
& =\sigma_{1}(X \cdot(1-\mathbb{E}) A)
\end{aligned}
$$

since $S_{1}(X \cdot(1-\mathbb{E}) A)=0$.

2.5 Representation theory of $\mathcal{D}_{n}^{(0)}$

Now that we know that $\mathcal{D}_{n}^{(0)}$ is unital, we can investigate its representation theory. We first look at the idempotents. 


\subsubsection{Idempotents in $\mathcal{D}_{n}^{(0)}$}

Recall that the (right) Zassenhaus idempotents $\zeta_{n}$ are defined as the homogeneous elements of degree $n$ (that is, $\zeta_{n} \in \mathbf{S y m}_{n}$ ) satisfying (see [19]):

$$
\sigma_{1}=: \prod_{k \geq 1} e^{\zeta_{k}}=e^{\zeta_{1}} e^{\zeta_{2}} e^{\zeta_{3}} \ldots
$$

Obviously, $\zeta_{1}=S_{1}$ so that $\zeta_{1}^{\sharp}=0$. Since the $\sharp$-transform is multiplicative,

$$
\sigma_{1}^{\sharp}=e^{\zeta_{2}^{\#}} e^{\zeta_{3}^{\sharp}} \ldots
$$

but also

$$
\sigma_{1}^{\sharp}=e^{-\zeta_{1}} \sigma_{1}=e^{\zeta_{2}} e^{\zeta_{3}} \ldots
$$

so that $\zeta_{i}^{\sharp}=\zeta_{i}$. Extracting the term of degree $n$, we have

$$
S_{n}^{\sharp}=\sum_{\substack{|\lambda|=n \\ 1 \notin \lambda}} \frac{\zeta^{\lambda}}{m_{\lambda}},
$$

where for a partition $\lambda=\left(\lambda_{1} \geq \lambda_{2} \geq \cdots\right), \zeta^{\lambda}:=\zeta_{\lambda_{1}} \cdots \zeta_{\lambda_{r}}$, and $m_{\lambda}:=\prod_{i \geq 1} m_{i}(\lambda)$ !, where $m_{i}(\lambda)$ the multiplicity of $i$ in $\lambda$.

We shall make use of the notation $e_{\lambda}:=\frac{\zeta^{\lambda}}{m_{\lambda}}$. For a composition $I$, we set $\zeta^{I}=$ $\zeta_{i_{1}} \ldots \zeta_{i_{r}}$ and $m_{I}=m_{\lambda}$ if $I \downarrow=\lambda$.

\subsubsection{A basis of idempotents}

The $\zeta_{n}$ are primitive elements with commutative image $p_{n} / n$, hence are Lie idempotents [19]. As with any sequence of Lie idempotents, we can construct an idempotent basis of $\mathbf{S y m}_{n}$ from the $\zeta_{n}$.

We need the following lemma from [24], easily derived from the splitting formula (compare [19, Lemma 3.10]).

Recall that the radical of $\left(\mathbf{S y m}_{n}, *\right)$ is $\mathcal{R}_{n}=\mathcal{R} \cap \mathbf{S y m}_{n}$, where $\mathcal{R}$ is the kernel of the commutative image $\mathbf{S y m} \rightarrow$ Sym.

Lemma 2.3 Denote by $\mathfrak{S}(J)$ the set of distinct rearrangements of a composition $J$. Let $I=\left(i_{1}, \ldots, i_{r}\right)$ and $J=\left(j_{1}, \ldots, j_{s}\right)$ be two compositions of $n$. Then

(i) if $\ell(J)<\ell(I)$ then $\zeta^{I} * \zeta^{J}=0$.

(ii) if $\ell(J)>\ell(I)$ then $\zeta^{I} * \zeta^{J} \in \operatorname{Vect}\left\langle\zeta^{K}: K \in \mathfrak{S}(J)\right\rangle \cap \mathcal{R}$. More precisely,

$$
\zeta^{I} * \zeta^{J}=\sum_{\substack{J_{1}, \ldots J_{r} \\\left|J_{k}\right|=i_{k}}}\left\langle J, J_{1} \sqcup \cdots \sqcup J_{r}\right\rangle \Gamma_{J_{1}} \cdots \Gamma_{J_{r}}
$$

where for a composition $K$ of $k, \Gamma_{K}:=\zeta_{k} * \zeta^{K}$, and $\amalg$ denotes the shuffle products of compositions regarded as words over the positive integers. 
(iii) if $\ell(J)=\ell(I)$, then $\zeta^{I} * \zeta^{J} \neq 0$ only for $J \in \mathfrak{S}(I)$, in which case $\zeta^{I} * \zeta^{J}=$ $m_{I} \zeta^{I}$

\section{Corollary 2.4}

(i) The elements

$$
e_{I}=\frac{1}{m_{I}} \zeta^{I}, \quad I \vDash n,
$$

are all idempotents and form a basis of $\mathbf{S y m}_{n}$. This basis contains in particular a complete set of minimal orthogonal idempotents, $e_{\lambda}$ of $\mathbf{S y m}_{n}$.

(ii) The $e_{I}$ such that I does not have a part equal to 1 form a basis of $\mathcal{D}_{n}^{(0)}$.

(iii) The $e_{\lambda}$ with no part equal to 1 in $\lambda$ form a complete set of minimal orthogonal idempotents of $\mathcal{D}_{n}^{(0)}$.

2.6 Cartan invariants of $\mathcal{D}_{n}^{(0)}$

By (iii) of Lemma 2.3, the indecomposable projective module $P_{\lambda}=\mathcal{D}_{n}^{(0)} * e_{\lambda}$ contains the $e_{I}$ for $I \in \mathfrak{S}(\lambda)$. For $I \notin \mathfrak{S}(\lambda)$, (i) and (ii) imply that $e_{I} * e_{\lambda}$ is in $\operatorname{Vect}\left\langle\zeta^{K}\right.$ : $K \in \mathfrak{S}(\lambda)\rangle$. Hence, this space coincides with $P_{\lambda}$. So, we get immediately an explicit decomposition

$$
\mathcal{D}_{n}^{(0)}=\bigoplus_{\lambda \vdash n, 1 \notin \lambda} P_{\lambda}, \quad P_{\lambda}=\bigoplus_{I \in \mathfrak{S}(\lambda)} \mathbb{C} e_{I} .
$$

The Cartan invariants

$$
c_{\lambda, \mu}=\operatorname{dim}\left(e_{\mu} * \mathcal{D}_{n}^{(0)} * e_{\lambda}\right)
$$

are now easily obtained. The above space is spanned by the

$$
e_{\mu} * e_{I} * e_{\lambda}=e_{\mu} * e_{I}, \quad I \in \mathfrak{S}(\lambda)
$$

From (ii) of Lemma 2.3, this space has the dimension of the space $\left[S^{\mu}(L)\right]_{\lambda}$, spanned by all symmetrized products of Lie polynomials of degrees $\mu_{1}, \mu_{2}, \ldots$ formed from $\zeta_{i_{1}}, \zeta_{i_{2}}, \ldots$, as in the classical result of Garsia-Reutenauer for the descent algebra [15].

In the following examples, partitions are ordered by reverse lexicographic order. For $n \leq 4$, the Cartan matrix of $\mathcal{D}_{n}^{0}$ is trivial: it is the identity matrix of size $d_{n}^{(0)}$, since there is at most one value in a partition of $n$ with no part one. For $n$ up to 7, the Cartan invariants are given by the matrices (60) and (61) at $q=1$. Indeed, the $q$-analogues defined from the Loewy series can be explicitly calculated.

\subsection{Quiver and $q$-Cartan invariants (Loewy series)}

We shall use the following modified refinement order on partitions: we write $\lambda \prec_{p} \mu$, and say that $\lambda$ is $p$-finer than $\mu$, if each part of $\mu$ is either a part of $\lambda$ or a sum of distinct parts of $\lambda$. Hence, $\mu$ covers $\lambda$ iff $\mu$ is obtained from $\lambda$ by merging two distinct parts. 
Still relying upon point (ii) of the lemma, we see that $c_{\lambda, \mu}=0$ if $\lambda$ is not $p$-finer than (or equal to) $\mu$, and that if $\mu$ is obtained from $\lambda$ by adding up two parts $\lambda_{i}, \lambda_{j}$, $e_{\mu} * e_{I}=0$ if $\lambda_{i}=\lambda_{j}$ and is a nonzero element of the radical otherwise.

The $q$-analogues of the Cartan invariants

$$
c_{\lambda, \mu}:=\sum_{k} q^{k} \operatorname{dim}\left[\left(e_{\mu} * \operatorname{rad}^{k} \mathcal{D}_{n}^{(0)} * e_{\lambda}\right) / \operatorname{rad}^{k+1} \mathcal{D}_{n}^{(0)}\right]
$$

can now be obtained from Proposition 2.2 and the following lemma.

Lemma 2.5 Let $A$ be an associative algebra. If $e$ is an idempotent of $A$ such that $e$ is neutral in $B=A e$, then

$$
\operatorname{rad}^{k} B=e\left(\operatorname{rad}^{k} A\right) e .
$$

Proof Let $x \in \operatorname{rad} B$. There exists an integer $n$ such that $(x B)^{n}=0$. Then

$$
(x e A e)^{n}=(x A)^{n} e=0,
$$

so that, as well

$$
(x A)^{n} \text { exA }=(x A)^{n} x A=(x A)^{n+1}=0 .
$$

Thus, the right ideal $x A$ is nilpotent, which proves that $x \in \operatorname{rad} A$. Since $x=$ exe, $x \in e \operatorname{rad} A e$ and we have shown that $\operatorname{rad} B \subseteq e \operatorname{rad} A e$.

Conversely, if $x \in \operatorname{rad} A$, so that $x^{n}=0$ for a certain $n$, then $(e x e)^{n}=x^{n} e=0$, whence exe $\in \operatorname{rad} B$, which proves the claim for $k=1$.

Now, if $x \in \operatorname{rad}^{k} B, x=x_{1} \ldots x_{k}$ with $x_{i}=e y_{i} e$, for some $y_{i} \in \operatorname{rad} A$. Hence,

$$
x=e y_{1} e \ldots e y_{k} e=e y_{1} y_{2} \ldots y_{k} e \in e \operatorname{rad}^{k} A e .
$$

Conversely, any $x$ of the form $e y_{1} \ldots y_{r} e$ with $y_{i} \in \operatorname{rad} A$ can be rewritten as $x=$ $e y_{1} e \ldots e y_{k} e \in \operatorname{rad}^{k} B$.

Applying this to $A=\mathbf{S y m}_{n}$, and $e=S_{n}^{\sharp}$, we obtain from the known description of the $q$-Cartan matrices of $\mathbf{S y m}_{n}[31]$ :

\section{Theorem 2.6}

(i) In the quiver of $\mathcal{D}_{n}^{(0)}$, there is an arrow $\lambda \rightarrow \mu$ iff $\mu$ is obtained from $\lambda$ by adding two distinct parts.

(ii) The q-Cartan invariants of $\mathcal{D}_{n}^{(0)}$ are given by

$$
c_{\lambda, \mu}(q)=c_{\lambda, \mu} q^{\ell(\lambda)-\ell(\mu)} .
$$

if $\lambda$ is finer than or equal to $\mu$, and $c_{\lambda, \mu}(q)=0$ otherwise.

The result can also be derived as follows: In [4], it is shown that the powers of the radical of $\mathbf{S y m}_{n}$ for the internal product coincide with the homogeneous component 
of degree $n$ of the lower central series of Sym for the external product:

$$
\mathcal{R}^{* j}=\gamma^{j}(\mathbf{S y m})
$$

where $\gamma^{j}(\mathbf{S y m})$ is the ideal of Sym generated by the commutators [Sym, $\left.\gamma^{j-1}(\mathbf{S y m})\right]$.

Since $\mathcal{D}^{(0)}$ is a free associative algebra over a sequence of primitive elements $\left(\zeta_{k}\right)_{k \geq 2}$ with the same internal product as in Sym, the argument of [4] can be reproduced verbatim, and we see that

$$
\left(\operatorname{rad} \mathcal{D}^{(0)}\right)^{* j}=\gamma^{j}\left(\mathcal{D}^{(0)}\right)=\mathcal{R}^{* j} \cap \mathcal{D}^{(0)}
$$

This shows that, in $\mathcal{D}^{(0)}$ as well as in $\mathbf{S y m}$, for $\lambda$ finer than $\mu, e_{\mu} * e_{I}$ is nonzero modulo $\operatorname{rad}^{* 2}$ iff $\mu$ is obtained from $\lambda$ by summing two distinct parts. And more generally, $e_{\mu} * e_{I}$ is in $\operatorname{rad}^{* k}$ and nonzero modulo $\operatorname{rad}^{* k+1}$ iff $\ell(\lambda)-\ell(\mu)=k$.

\subsection{Tables of the $q$-Cartan invariants of $\mathcal{D}_{n}^{(0)}$}

The labels for row and columns of the $q$-Cartan matrices, namely partitions with no part one, are in reverse lexicographic order. In the following matrices, the zero entries are represented by dots to enhance readability.

For $n \leq 4$, the $q$-Cartan matrix of $\mathcal{D}_{n}^{(0)}$ is the identity matrix of size $d_{n}^{(0)}$. The first non-trivial example arises for $n=5$. The $q$-Cartan matrices of $\mathcal{D}_{5}^{(0)}, \mathcal{D}_{6}^{(0)}$, and $\mathcal{D}_{7}^{(0)}$ are respectively

$$
\left(\begin{array}{ll}
1 & q \\
. & 1
\end{array}\right) \quad\left(\begin{array}{cccc}
1 & q & \cdot & . \\
\cdot & 1 & . & . \\
\cdot & \cdot & 1 & . \\
\cdot & \cdot & . & 1
\end{array}\right) \quad\left(\begin{array}{cccc}
1 & q & q & q^{2} \\
\cdot & 1 & \cdot & q \\
\cdot & \cdot & 1 & \cdot \\
\cdot & \cdot & . & 1
\end{array}\right)
$$

and those of $\mathcal{D}_{8}^{(0)}$ and $\mathcal{D}_{9}^{(0)}$ are

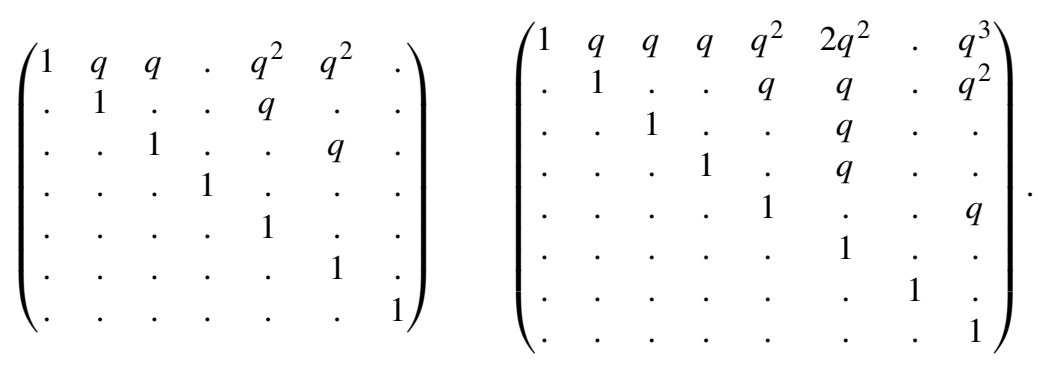

On these matrices, one can read the quiver of $\mathcal{D}_{n}^{(0)}$. Note that it is a subquiver of the quiver of $\mathbf{S y m}_{n}$ (see [31]), since one cannot create parts 1 by merging parts of non-unitary partitions. 
2.9 The (large) derangement algebra $\mathcal{D}=\mathcal{D}^{(\infty)}$

Pursuing the analogy with the peak algebra, let us define

$$
\mathcal{D}:=\bigoplus_{k \geq 0} S_{k} \mathcal{D}^{(0)}
$$

Note already that $\mathcal{D}$ is not a subalgebra of $\mathbf{S y m}$. It is only a sub-coalgebra. Moreover, we have the following theorem.

Theorem 2.7 Each homogeneous component $\mathcal{D}_{n}$ of $\mathcal{D}$ is stable under $*$. It is a unital algebra, since it contains $S_{n}$, the neutral element of $*$ in $\mathbf{S y m}$.

We shall prove a slightly more general result, interpolating between $\mathcal{D}^{(0)}$ and $\mathcal{D}$.

\subsection{A filtration of $\mathcal{D}$}

Define $\mathcal{D}_{n}^{(k)}$ by

$$
\mathcal{D}_{n}^{(k)}:=\bigoplus_{j=0}^{k} S_{j} \mathcal{D}_{n-j}^{(0)}
$$

For $k=0$, this is $\mathcal{D}_{n}^{(0)}$ and for $k \geq n$, one recovers $\mathcal{D}_{n}$.

The following alternative definition of the filtration will be useful in the sequel:

\section{Lemma 2.8}

$$
\mathcal{D}_{n}^{(k)}=\bigoplus_{j=0}^{\min (n, k)} S_{1}^{j} \mathcal{D}_{n-j}^{(0)} .
$$

Proof Expanding $\sigma_{1}=e^{S_{1}} \sigma_{1}^{\sharp}$, we see that

$$
S_{k} \equiv \frac{S_{1}^{k}}{k !} \quad\left(\bmod \bigoplus_{j<k} S_{j} \mathcal{D}_{n-j}^{(0)}\right) .
$$

2.11 Dimensions of the $\mathcal{D}_{n}^{(k)}$

From (63), we see that the dimension $d_{n}^{(k)}$ of $\mathcal{D}_{n}^{(k)}$ is

$$
d_{n}^{(k)}=\sum_{i \leq k} d_{n-i}^{(0)} .
$$

For $k=\infty$, these are the usual Fibonacci numbers.

The first values are given in Table 1 . 
Table 1

\begin{tabular}{lllllllllll}
\hline$n$ & 0 & 1 & 2 & 3 & 4 & 5 & 6 & 7 & 8 & 9 \\
\hline$d_{n}^{(0)}$ & 1 & 0 & 1 & 1 & 2 & 3 & 5 & 8 & 13 & 21 \\
$d_{n}^{(1)}$ & 1 & 1 & 1 & 2 & 3 & 5 & 8 & 13 & 21 & 34 \\
$d_{n}^{(2)}$ & 1 & 1 & 2 & 2 & 4 & 6 & 10 & 16 & 26 & 42 \\
$d_{n}^{(3)}$ & 1 & 1 & 2 & 3 & 4 & 7 & 11 & 18 & 29 & 47 \\
$d_{n}^{(\infty)}$ & 1 & 1 & 2 & 3 & 5 & 8 & 13 & 21 & 34 & 55 \\
\hline
\end{tabular}

2.12 The complete picture

Theorem 2.9 For all $n$ and $k$, each homogeneous component $\mathcal{D}_{n}^{(k)}$ of $\mathcal{D}^{(k)}$ is stable under $*$. It is a unital algebra and the neutral element is

$$
P_{n}^{(k)}:=\sum_{i=0}^{\min (k, n)} \frac{S_{1}^{i}}{i !} S_{n-i}^{\sharp} .
$$

The theorem is a consequence of the following lemma.

Lemma 2.10 Let $f$ and $g$ be in Sym. Then

$$
\left(\frac{S_{1}^{m}}{m !} f^{\sharp}\right) *\left(\frac{S_{1}^{n}}{n !} g^{\sharp}\right)= \begin{cases}0 & \text { if } m \neq 0, \\ \frac{S_{1}^{n}}{n !}\left(f^{\sharp} * g^{\sharp}\right) & \text { otherwise. }\end{cases}
$$

Proof Replacing the right factor by its generating series, we have

$$
\begin{aligned}
\left(S_{1}^{m} f^{\sharp}\right) *\left(e^{S_{1}} g^{\sharp}\right) & =\sum_{(g)}\left(S_{1}^{m} *\left(e^{S_{1}} g_{(1)}^{\sharp}\right)\right)\left(f^{\sharp} *\left(e^{S_{1}} g_{(2)}^{\sharp}\right)\right) \\
& =\sum_{(g)}\left(e^{S_{1}} g_{(1)}^{\sharp} * S_{1}^{m}\right)\left(f^{\sharp} *\left(e^{S_{1}} g_{(2)}^{\sharp}\right)\right),
\end{aligned}
$$

since $S_{1}^{m}$ is central for $*$ Now, $\left(e^{S_{1}} S^{I^{\sharp}} * S_{1}^{m}\right)=0$ if $I$ is not empty since $S_{n}^{\sharp} * S_{1}^{n}=$ $S_{1}^{n} * S_{n}^{\sharp}=0$ for $n \geq 1$ as $e^{-S_{1}} * \sigma_{1}^{\sharp}=1$. So, the whole sum reduces to

$$
S_{1}^{m}\left(f^{\sharp} *\left(e^{S_{1}} g^{\sharp}\right)\right) .
$$

Thus,

$$
f^{\sharp} *\left(e^{S_{1}} g^{\sharp}\right)=f * \sigma_{1}^{\sharp} *\left(e^{S_{1}} g^{\sharp}\right) .
$$

Consider now the generic case $g=\sigma_{1}(X A)$ :

$$
\begin{aligned}
\sigma_{1}^{\sharp} *\left(e^{S_{1}} \sigma_{1}(X A)^{\sharp}\right) & =\left(e^{-S_{1}} \sigma_{1}\right) *\left(e^{S_{1}} \sigma_{1}(X A)^{\sharp}\right) \\
& =\left(e^{-S_{1}} * e^{S_{1}} \sigma_{1}(X A)^{\sharp}\right)\left(e^{S_{1}} \sigma_{1}(X A)^{\sharp}\right)
\end{aligned}
$$




$$
\begin{aligned}
& =\left(e^{S_{1}} * e^{-S_{1}}\right)\left(\sigma_{1}(X A)^{\sharp} * e^{-S_{1}}\right)\left(e^{S_{1}} \sigma_{1}(X A)^{\sharp}\right) \\
& =e^{-S_{1}}\left(e^{-S_{1}} * \sigma_{1}(X A)^{\sharp}\right)\left(e^{S_{1}} \sigma_{1}(X A)^{\sharp}\right) \\
& =e^{-S_{1}} e^{-S_{1}(X A)^{\sharp}} e^{S_{1}} \sigma_{1}(X A)^{\sharp} \\
& =e^{-S_{1}} e^{S_{1}} \sigma_{1}(X A)^{\sharp}=\sigma_{1}(X A)^{\sharp}
\end{aligned}
$$

where the third and fourth equalities come from the fact that $e^{-S_{1}}$ is central for $*$ and idempotent. Multiplying by $f$ on the left yields

$$
f^{\sharp} *\left(e^{S_{1}} g^{\sharp}\right)=f * g^{\sharp},
$$

whence the statement.

Proof of the theorem From Lemma 2.8, we have $P_{n}^{(k)} \in \mathcal{D}_{n}^{(k)}$. Moreover, since the $S_{1}^{k} S^{I \sharp}$ with $I$ nonunitary form a basis of $\mathcal{D}_{n}^{(k)}$ adapted to the direct sum decomposition, that $P_{n}^{(k)}$ is neutral on both sides is equivalent to the already known fact that $S_{n}^{\sharp}$ is neutral in $\mathcal{D}_{n}^{(0)}$.

Corollary 2.11 The map $\phi_{m}: \mathcal{D}_{k}^{(0)} \rightarrow \mathcal{D}_{k+m}^{(m)}$ defined by

$$
\phi_{m}(f)=\frac{S_{1}^{m}}{m !} f
$$

is a (nonunital) monomorphism of algebras.

Corollary 2.12 As an algebra, $\mathcal{D}_{n}$ is isomorphic to the direct sum

$$
\mathcal{D}_{n} \sim \bigoplus_{k=0}^{n} \mathcal{D}_{n-k}^{(0)}
$$

Proof As a vector space, $\mathcal{D}_{n}$ is the direct sum of the spaces $V_{n}^{(k)}=S_{1}^{k} \mathcal{D}_{n-k}^{(0)}$. By Lemma $2.10, V_{n}^{(k)} * V_{n}^{(\ell)}=0$ for $k \neq \ell$, and by Corollary 2.11 , each $V_{n}^{(k)}$ is a subalgebra isomorphic to $D_{n-k}^{(0)}$.

2.13 Representation theory of $\mathcal{D}_{n}^{(k)}$

We are now in a position to deduce the representation theory of the $\mathcal{D}_{n}^{(k)}$ from that of $\mathcal{D}_{n}^{(0)}$. We can extend Corollary 2.4 to all values of $k$, as a direct consequence of Lemma 2.3.

Corollary 2.13 Let $e_{I}$ be the idempotents of $\mathbf{S y m}$ defined in Corollary 2.4.

(i) The $e_{J}$ such that $J=\left(1^{j}, I\right)$ with $j \leq k$ and I does not contain a part 1 form a basis of $\mathcal{D}_{n}^{(k)}$.

(ii) The principal idempotents of $\mathcal{D}_{n}^{(k)}$ are the $e_{\lambda}$ such that $m_{1}(\lambda) \leq k$. 
We can now state the general result on the representation theory of $\mathcal{D}_{n}^{(k)}$ :

Corollary 2.14 The irreducible representations of $\mathcal{D}_{n}^{(k)}$ are of dimension 1 and parameterized by partitions of $n$ with at most $k$ parts equal to 1 .

Note that the principal idempotents of $\mathcal{D}_{n}$ are also a complete set of minimal orthogonal idempotents of $\mathbf{S y m}_{n}$.

\section{$2.14 q$-Cartan matrix of $\mathcal{D}_{n}^{(k)}$}

We order the labels of the rows and columns of the $q$-Cartan matrices, namely partitions with at most $k$ ones, first by their number of ones and then in reverse lexicographic order. So, for example, with $n=5$ and $k=3$, the order is

$$
[5,32,41,221,311,2111] \text {. }
$$

With this convention, the $q$-Cartan matrix of $\mathcal{D}_{n}^{(k)}$ is the block-diagonal matrix obtained by putting on the diagonal the $q$-Cartan matrices of $\mathcal{D}_{n-i}^{(0)}$ for $i \leq \min (k, n)$. Indeed, from the previous results, one easily sees that

\section{Lemma 2.15}

$$
\mathcal{D}_{n}^{(k)}=P_{n}^{(k)} * \mathbf{S y m}_{n} * P_{n}^{(k)}
$$

Since each $P_{n}^{(k)}$ is a sum of orthogonal idempotents (the $S_{1}^{i} S_{n-i}^{\sharp} / i$ !), this proves that the $q$-Cartan matrix of $\mathcal{D}_{n}^{(\infty)}$ is deduced from the $q$-Cartan matrix of $\mathbf{S y m}_{n}$ by putting to 0 the entries whose row and column do not have the same number of ones.

\subsection{Block projectors}

We have seen that each space $S_{1}^{j} \mathcal{D}_{n-j}^{(0)}$ has as unit a part of $P_{n}^{(k)}$, namely

$$
D_{n, k}=\frac{S_{1}^{k}}{k !} S_{n-k}^{\sharp} .
$$

Their double generating series is

$$
D(t, u ; A):=\sum_{n \geq 0} \sum_{k=0}^{n} t^{n-k} u^{k} D_{n, k}(A)=e^{(u-t) S_{1}(A)} \sigma_{t}(A),
$$

that is, the noncommutative analog of the generating function of $d_{n, k}$, the number of permutations in $\mathfrak{S}_{n}$ with exactly $k$ fixed points:

$$
D(t, u)=\sum_{n \geq 0} \frac{1}{n !} \sum_{k=0}^{n} d_{n, k} u^{k} t^{n-k}=\frac{e^{u-t}}{1-t} .
$$




\section{$2.16 q$-dimension polynomials}

If one sums up the entries of the $q$-Cartan matrix of $\mathcal{D}_{n}^{(\infty)}$, one gets the following polynomials in $q$, refining the Fibonacci numbers:

$$
1,2,3,5, q+7,2 q+11, q^{2}+5 q+15,3 q^{2}+9 q+22, \ldots
$$

better represented in the following triangle:

$\begin{array}{ccccccc}1 & & & & & & \\ 2 & & & & & & \\ 3 & & & & & & \\ 5 & & & & & & \\ 7 & 1 & & & & & \\ 11 & 2 & & & & & \\ 15 & 5 & 1 & & & & \\ 22 & 9 & 3 & & & & \\ 30 & 17 & 7 & 1 & & & \\ 42 & 28 & 16 & 3 & & & \\ 56 & 47 & 31 & 9 & 1 & & \\ 77 & 73 & 58 & 21 & 4 & & \\ 101 & 114 & 102 & 47 & 12 & 1 & \\ 135 & 170 & 175 & 94 & 32 & 4 & \\ 176 & 253 & 286 & 183 & 74 & 14 & 1 \\ 231 & 365 & 461 & 333 & 162 & 40 & 5\end{array}$

The first column (constant terms of the polynomials) corresponds to the size of the matrix, hence the number of partitions of $n$. The second column is sequence A000097 of [35]: from the characterization of the quiver of $\mathbf{S y m}_{n}$, we also have that the second column gives to the number of ways of selecting two different parts different from 1 , in all partitions of $n$. Finally, it is also equal to the number of ways of selecting two different parts, in all partitions of $n-2$, hence justifying that the number of arrows in the quiver of $\mathcal{D}_{n}^{(\infty)}$ is equal to the number of arrows in the quiver of $\mathbf{S y m}_{n-2}$.

\section{The $(1-\mathbb{E})$ transform in WQSym*}

\subsection{Word quasi-symmetric functions}

A word $u$ over $\mathbb{N}^{*}$ is said to be packed if the set of letters occurring in $u$ is an interval of $\mathbb{N}^{*}$ containing 1 . The algebra $\operatorname{WQSym}(A)$ (Word Quasi-Symmetric functions) is defined as the subalgebra of $\mathbb{K}\langle A\rangle$ based on packed words and spanned by the elements

$$
\mathbf{M}_{u}(A):=\sum_{\operatorname{pack}(w)=u} w
$$


where $\operatorname{pack}(w)$ is the packed word of $w$, that is, the word obtained by replacing all occurrences of the $k$-th smallest letter of $w$ by $k$. For example,

$$
\operatorname{pack}(871883319)=431442215
$$

This is the invariant algebra of the quasi-symmetrizing action of $\mathfrak{S}(A)$ on $\mathbb{K}\langle A\rangle$ [10]. Packed words can be identified with set compositions in an obvious way, and geometrically, they can be interpreted as facets of the permutohedron: a packed word $w=w_{1} \ldots w_{n}$ with largest entry $\ell$ can be identified with the set composition $\left[P_{1}, \ldots, P_{\ell}\right]$ where $P_{j}=\left\{i \leq n \mid w_{i}=j\right\}$. For example, 431442215 corresponds to $[\{3,8\},\{6,7\},\{2\},\{1,4,5\},\{9\}]$.

Let $\mathbf{N}_{u}=\mathbf{M}_{u}^{*}$ be the dual basis of $\left(\mathbf{M}_{u}\right)$. It is known that WQSym is a self-dual Hopf algebra $[18,27]$ and that on the graded dual WQSym*, an internal product * may be defined by

$$
\mathbf{N}_{u} * \mathbf{N}_{v}=\mathbf{N}_{\operatorname{pack}(u, v)},
$$

where the packing of biwords is defined with respect to the lexicographic order on biletters, so that, for example,

$$
\operatorname{pack}\left(\begin{array}{l}
42412253 \\
53154323
\end{array}\right)=62513274 .
$$

This product is induced from the internal product of parking functions [25, 26, 28] and allows one to identify the homogeneous components $\mathbf{W Q S y m}_{n}$ with the (opposite) Solomon-Tits algebras, in the sense of [30].

The (opposite) Solomon descent algebra, realized as $\mathbf{S y m}_{n}$, is embedded in the (opposite) Solomon-Tits algebra realized as WQSym* by

$$
S^{I}=\sum_{\mathrm{ev}(u)=I} \mathbf{N}_{u}
$$

where $\operatorname{ev}(u)$ is the evaluation of $u$, i.e., the vector whose $i$ th component is the number of occurrences of $i$ in $u$.

From now on, we shall denote WQSym* by $\mathcal{W}$.

\subsection{Idempotents of $\mathcal{W}$}

\subsubsection{The semisimple quotient}

It is known that the radical of $\mathcal{W}_{n}$ is spanned by the differences

$$
\mathbf{N}_{u}-\mathbf{N}_{v}
$$

where $v=\sigma(u)$ for some permutation $\sigma$ of the support of $u, \operatorname{supp}(u)=\left\{\left.i|| u\right|_{i} \neq 0\right\}$. This is easily seen: Equation (85) implies that the $\mathbf{N}_{u}-\mathbf{N}_{v}$ are nilpotent of order 2 if $v=\sigma(u)$, and that their span is an ideal $\mathcal{R}_{n}$. Moreover, any product of $n$ such factors $\mathbf{N}_{u_{i}}-\mathbf{N}_{v_{i}}$ vanishes since the product of two such factors is either strictly finer than $u_{i}$ or zero, so that $\mathcal{R}_{n}$ is nilpotent. The quotient $W_{n} / \mathcal{R}_{n}$ is semisimple. It 
can be identified with the (commutative) algebra of set partitions with $\wedge$ (the inf for the refinement order on set partitions) as product. Indeed, packed words encode set compositions, $u=u_{1} \ldots u_{n}$ corresponding to the set composition of $[n]$ in which $i$ belongs to the block $u_{i}$, e.g.,

$$
u=21231 \Longleftrightarrow[\{2,5\},\{1,3\},\{4\}],
$$

and the left action of permutations amounts to permuting the blocks, e.g., with $\sigma=231$,

$$
\sigma(21231)=32312 \Longleftrightarrow[\{4\},\{2,5\},\{1,3\}] .
$$

Hence, the idempotents of a complete family of $\mathcal{W}_{n}$ are parametrized by set partitions of $[n]$.

\subsubsection{The idempotents of Saliola}

In [31], Saliola has given a general recipe for constructing such complete sets. Given a packed word $u$, denote by $\Pi(u)$ the set partition obtained by forgetting the order among the blocks of the set compositions encoded by $u$.

For each set partition $\pi$ of $[n]$, choose a linear combination

$$
l_{\pi}=\sum_{\Pi(u)=\pi} c_{u} \mathbf{N}_{u}
$$

where the coefficient $c_{u}$ depends only on the evaluation $\operatorname{ev}(u)$ of $u$, and

$$
\sum_{\Pi(u)=\pi} c_{u}=1
$$

Start with the initial condition

$$
e_{\{1\},\{2\}, \ldots,\{n\}}=\frac{1}{n !} \sum_{\sigma \in \mathfrak{S}_{n}} \mathbf{N}_{\sigma},
$$

hence equal to $S_{1}^{n} / n$ ! in $\mathbf{S y m}$, and define by the induction

$$
e_{\pi}=l_{\pi} *\left(\mathbf{N}_{1^{n}}-\sum_{\pi^{\prime}>\pi} e_{\pi^{\prime}}\right)
$$

where $\mathbf{N}_{1^{n}}=S_{n}$ is the identity of $*$, and $\geq$ is the reverse refinement order. Then the $e_{\pi}$ form a complete set of orthogonal idempotents of $\mathcal{W}_{n}$.

\subsubsection{A nonrecursive construction}

Families of Saliola idempotents can be computed for all $\mathcal{W}_{n}$ simultaneously, in a nonrecursive way from families of idempotents of the descent algebra $\mathbf{S y m}_{n}$ constructed by the method developed in [19]. Recall that the starting point of this construction is 
a sequence of Lie idempotents $\gamma_{n} \in \mathbf{S y m}_{n}$, that is, an arbitrary sequence of primitive elements whose commutative image in $S y m$ is $p_{n} / n$.

Then if we decompose the identity $S_{n}$ of $\mathbf{S y m}_{n}$ as

$$
S_{n}=\sum_{I \vDash n} c_{I} \gamma^{I}
$$

the elements

$$
e_{\lambda}:=\sum_{I \downarrow \lambda} c_{I} \gamma^{I}
$$

where $I \downarrow \lambda$ means that the nondecreasing rearrangement of the composition $I$ is the partition $\lambda$, with $\lambda$ a partition of $n$, form a complete family of orthogonal idempotents of $\mathbf{S y m}_{n}$.

Let us fix such a family, and define for each set partition $\pi$ of $[n]$

$$
l_{\pi}=\sum_{\Pi(u)=\pi} c_{\mathrm{ev}(u)} \mathbf{N}_{u} .
$$

These elements satisfy Saliola's conditions: obviously, $c_{\mathrm{ev}(u)}$ depends only on ev $(u)$, and

$$
\sum_{\Pi(u)=\pi} c_{\mathrm{ev}(u)}=\prod_{i} m_{i}(\lambda) ! \sum_{I \downarrow \lambda} c_{I}
$$

is the coefficient of $p_{\lambda} / z_{\lambda}$ in the commutative image of $S_{n}$, which is $h_{n}$, so it is 1 . Hence, the sequence $\left(\gamma_{n}\right)$ determines idempotents $e_{\pi}$ of the $\mathcal{W}_{n}$ by the recursion (94). But we can also compute these directly as follows.

Theorem 3.1 The idempotents $e_{\pi}$ are given by the internal products

$$
e_{\pi}=l_{\pi} * e_{\lambda}
$$

Proof Let $\tilde{e}_{\pi}=l_{\pi} * e_{\lambda}$. For $\pi=\{\{1\}, \ldots,\{n\}\}$, we have $l_{\pi}=S_{1}^{n} / n !, e_{\lambda}=S_{1}^{n} / n$ !, so that $\tilde{e}_{\pi}=S_{1}^{n} / n !$.

Let $l_{\lambda}=\sum_{\Lambda(\pi)=\lambda} l_{\pi}$, where $\Lambda(\pi)$ is the integer partition recording the block lengths of $\pi$. We have

$$
l_{\lambda}=\sum_{I \downarrow \lambda} c_{I} S^{I} \equiv e_{\lambda} \quad \bmod \bigoplus_{l(I)>l(\lambda)} \mathbb{K} \gamma^{I},
$$

so that $e_{\lambda}=l_{\lambda} * e_{\lambda}$ in Sym.

We want to show that $\tilde{e}_{\pi}=e_{\pi}$. For that, recall from [31] that, if $\Pi(u) \not \leq \pi, \mathbf{N}_{u} *$ $e_{\pi}=0$, so that $l_{\pi} * e_{\pi^{\prime}}=0$ if $\pi \ngtr \pi^{\prime}$, and $\Lambda\left(\pi^{\prime}\right) \neq \Lambda(\pi)$. This implies in particular that $e_{\lambda}=\sum_{\Lambda(\pi)=\lambda} e_{\pi}$. Indeed, this is true for $\lambda=1^{n}$, and, by induction, $e_{\pi}=l_{\pi} *$ $\left(\mathbf{N}_{1^{n}}-\sum_{l\left(\pi^{\prime}\right)>l(\pi)} e_{\pi^{\prime}}\right)$, since $\pi^{\prime}>\pi$ implies $l\left(\pi^{\prime}\right)>l(\pi)$. Hence

$$
e_{\pi}=l_{\pi} *\left(\mathbf{N}_{1^{n}}-\sum_{l\left(\lambda^{\prime}\right)>l(\lambda)} e_{\lambda^{\prime}}\right)=l_{\pi} * \sum_{l\left(\lambda^{\prime}\right) \leq l(\lambda)} e_{\lambda^{\prime}} .
$$


Summing over $\pi$, we get

$$
\sum_{\Lambda(\pi)=\lambda} e_{\pi}=l_{\lambda} * \sum_{l\left(\lambda^{\prime}\right) \leq l(\lambda)} e_{\lambda^{\prime}}=e_{\lambda}
$$

Now,

$$
\begin{aligned}
e_{\pi} & =l_{\pi} *\left(\mathbf{N}_{1^{n}}-\sum_{\pi^{\prime}>\pi} e_{\pi^{\prime}}\right)=l_{\pi} * \sum_{\pi^{\prime} \ngtr \pi} e_{\pi^{\prime}} \\
& =l_{\pi} *\left(e_{\lambda}+\sum_{\pi^{\prime} \ngtr \pi ; \Lambda\left(\pi^{\prime}\right) \neq \Lambda(\pi)} e_{\pi^{\prime}}\right)=l_{\pi} * e_{\lambda}=\tilde{e}_{\pi} .
\end{aligned}
$$

\subsection{The $(1-\mathbb{E})$-transform in $\mathcal{W}$}

The embedding (87) of Sym in $\mathcal{W}$ can be defined on the generators as

$$
S_{n} \longmapsto \mathbf{N}_{1^{n}}
$$

It is clearly a bialgebra morphism. The element

$$
\sigma_{1}^{\sharp}=e^{-\mathbf{N}_{1}} \sum_{n \geq 0} \mathbf{N}_{1^{n}}
$$

is well defined in $\mathcal{W}$, and so is the $\sharp$-transform

$$
F^{\sharp}:=F * \sigma_{1}^{\sharp} \text {. }
$$

\subsection{Bases of $\mathcal{W}^{\sharp}$}

Let us say that a packed word $u$ is nonunitary (and unitary otherwise) if no letter occurs exactly once in $u$. These words correspond to set compositions without singletons.

Proposition 3.2 The $\mathbf{N}_{u}^{\sharp}$ with u nonunitary form a basis of $\mathcal{W}^{\sharp}$.

Proof Let us say that $v$ is finer than $u$ (and write $v>u$ ) if the set composition encoded by $v$ is finer than the set composition encoded by $u$. Then

$$
\mathbf{N}_{u} * \sigma_{1}^{\sharp}=\mathbf{N}_{u}+\sum_{v} c_{u v} \mathbf{N}_{v}
$$

where $v>u$ or $v$ is unitary. Hence, the $\mathbf{N}_{v}^{\sharp}$ with $u$ nonunitary are linearly independent. 


\subsection{Algebraic structure of $\mathcal{W}^{\sharp}$}

Let $\mathcal{J}$ be the two-sided ideal of $\mathcal{W}$ generated by the $\mathbf{N}_{u}$ such that $u$ has at least a letter occurring exactly once. The product rule (85) shows that $\mathcal{J}$ is an ideal for the internal product as well. Hence, the projection

$$
\pi: \mathcal{W} \longrightarrow \mathcal{W} / \mathcal{J}
$$

is a morphism for $*$. Its restriction to $\mathcal{W}^{\sharp}$ is then an isomorphism, and clearly,

$$
\pi\left(\sigma_{1}^{\sharp}\right)=\sigma_{1} .
$$

Since $\sigma_{1}$ is neutral in $\mathcal{W} / \mathcal{J}$, we have the following proposition.

Proposition $3.3 \sigma_{1}^{\sharp}$ is neutral in $\mathcal{W}^{\sharp}$.

Note that this proof would apply to Sym as well. To summarize, we have the following proposition.

Proposition 3.4 $\mathcal{W}^{\sharp}$ is isomorphic to $\mathcal{W} / \mathcal{J}$ as a Hopf algebra, and each $\mathcal{W}_{n}^{\sharp}$ is *-isomorphic to $\mathcal{W}_{n} / \mathcal{J}_{n}$, with $D_{n}=S_{n}^{\sharp}=\mathbf{N}_{1^{n}}^{\sharp}$ as neutral element.

\subsection{Representation theory of $\mathcal{W}^{\sharp}$}

We can now apply Lemma 2.5 with $A=\mathcal{W}_{n}, B=\mathcal{W}_{n}^{\sharp}$ and $e=\mathbf{S}_{n}^{\sharp}$.

Thus,

$$
\operatorname{rad}^{k} \mathcal{W}_{n}^{\sharp}=D_{n} *\left(\operatorname{rad}^{k} \mathcal{W}_{n}\right) * D_{n}
$$

The irreducible representations of $\mathcal{W}_{n}$, which are one-dimensional are parameterized by set partitions of $[n]$.

The $q$-Cartan matrices and quiver of $\mathcal{W}_{n}$ have been determined in [33]:

$$
c_{\alpha, \beta}(q)=c_{\alpha, \beta} q^{l(\alpha)-l(\beta)},
$$

where $l(\pi)$ is the number of blocks of a set partition $\pi$, and the Cartan invariant $c_{\alpha, \beta}$ is 0 if $\alpha$ is not finer than $\beta$, and otherwise

$$
c_{\alpha, \beta}=\prod_{i}\left(m_{i}-1\right) !,
$$

where for each block $B_{i}$ of $\beta, m_{i}$ is the number of blocks of $\alpha$ into which $B_{i}$ has been split.

For example, with $\alpha=12|3| 4|56| 7$ and $\beta=1234 \mid 567$, we have: $c_{\alpha, \beta}=$ $(3-1) !(2-1) !=2, l(\alpha)=5, l(\beta)=2$, so that $c_{\alpha, \beta}(q)=2 q^{3}$.

Theorem 3.5 The q-Cartan matrix of $\mathcal{W}_{n}^{\sharp}$ is the restriction to rows and columns indexed by nonunitary set partitions of $[n]$ of (111). In particular, the vertices of the quiver of $\mathcal{W}_{n}^{\sharp}$ are the non-unitary set partitions, and there is an arrow $\alpha \rightarrow \beta$ whenever $\beta$ is obtained from $\alpha$ by merging two blocks. 


\subsection{Analogue of $\mathcal{D}$ in $\mathcal{W}$}

Let $\mathcal{V}_{n}^{(0)}=\mathcal{W}_{n}^{\sharp}$ and

$$
\mathcal{V}_{n}^{(k)}=\bigoplus_{k=0}^{n} D_{n, k} * \mathcal{W}_{n} * D_{n, k}
$$

Then, as in the case of $\mathbf{S y m}$, each $\mathcal{V}_{n}^{(k)}$ is a unital subalgebra of $\mathcal{W}_{n}^{\sharp}$.

\section{The $(1-\mathbb{E})$-transform in FQSym}

\subsection{Definition}

Recall that FQSym is based on permutations, that in the mutually dual bases $\mathbf{F}_{\sigma}=$ $\mathbf{G}_{\sigma^{-1}}$, the internal product is defined by

$$
\mathbf{F}_{\sigma} * \mathbf{F}_{\tau}=\mathbf{F}_{\sigma \tau} \quad \text { or equivalently } \quad \mathbf{G}_{\sigma} * \mathbf{G}_{\tau}=\mathbf{G}_{\tau \sigma},
$$

and that Sym is embedded into FQSym by $S_{n}=\mathbf{G}_{12 \ldots n}$. The transformation can therefore be defined by

$$
\mathbf{F}_{\tau}^{\sharp}:=\mathbf{F}_{\tau} * \sigma_{1}^{\sharp} \text {. }
$$

Since the splitting formula remains valid in FQSym when the right factor of the internal product is in Sym [10], this is again a Hopf algebra morphism.

As we shall see below, in FQSym, the idempotent $D_{n}=S_{n}^{\sharp}$ as well as the other $D_{n, k}$ defined in (78) admit an interesting interpretation.

\subsection{The Tsetlin library (uniform case)}

The $(1-\mathbb{E})$-transform in FQSym is related to a classical problem in probability theory known as the Tsetlin library (see e.g., [2]). This is a Markov chain on $\mathfrak{S}_{n}$, defined by a shelf of $n$ books, which are randomly picked by users and then put back at the left of the shelf after use. In the uniform case (when all books are picked with the same probability), the determination of the stationary distribution amounts to the diagonalization of the linear operator on $\mathbb{C S}_{n}$

$$
t_{n}(f)=f \tau_{n}
$$

where

$$
\tau_{n}=12 \ldots n+2134 \ldots n+3124 \ldots n+\cdots+n 12 \ldots n-1 \in \mathbb{C S}_{n} .
$$

This problem is also an ingredient of the proof of Hivert's conjecture by Garsia and Wallach [16]. It can be solved in many different ways. The following one is quite natural in the context of Noncommutative Symmetric Functions.

We start with the observation that $\tau_{n}$ is in the descent algebra $\Sigma_{n}$. Indeed, $\tau_{n}=$ $D_{\subseteq\{1\}}$ (the sum of permutations having at most a descent at the first position), so 
that its representation as a noncommutative symmetric function is $S^{1, n-1}$, a rather well-understood element.

From this remark, we obtain immediately the eigenvalues of $t_{n}$. Indeed, according to Proposition 3.12 of [19], these are the scalar products $\left\langle h_{n-1} h_{1}, p_{\lambda}\right\rangle$ of ordinary symmetric functions. Clearly, the scalar product evaluates to $m_{1}(\lambda)$, so that the spectrum is $0,1,2, \ldots, n-2, n$.

Let us now construct the spectral projectors. To this aim, we shall need to evaluate some polynomials in $t_{n}$. Let us set $T_{n}=S^{1, n-1}$ and consider the generating function

$$
T=\sum_{n \geq 0} T_{n}=S_{1} \sigma_{1} .
$$

Since the internal products $T_{n} * T_{m}$ are (by definition) 0 for $m \neq n$, we have

$$
\sum_{n} T_{n}^{* r}=T^{* r}
$$

and using iteratively the splitting formula ([19], Proposition 2.1)

$$
T * T^{*(r-1)}=\left(S_{1} \sigma_{1}\right) * T^{*(r-1)}=\mu\left[\left(S_{1} \otimes \sigma_{1}\right) *_{2} \Delta\left(T^{*(r-1)}\right)\right],
$$

we get the expression

$$
T^{* r}=B_{r}\left(S_{1}\right) \sigma_{1},
$$

where $B_{r}(x)$ are the Bell polynomials (this is the obvious noncommutative analogue of the classical formula for the Kronecker powers of the representation of $\mathfrak{S}_{n}$ by permutation matrices).

Using the fact that the coefficients of $B_{n}$ are the Stirling numbers of the second kind $S(n, k)$, we obtain in Sym

$$
T *(T-1) *(T-2) * \cdots *(T-k+1)=S_{1}^{k} \sigma_{1},
$$

and in particular, in degree $n$,

$$
T_{n} *\left(T_{n}-1\right) *\left(T_{n}-2\right) * \cdots *\left(T_{n}-n+1\right)=S_{1}^{n}
$$

and as well

$$
T_{n} *\left(T_{n}-1\right) *\left(T_{n}-2\right) * \cdots *\left(T_{n}-n+2\right)=S_{1}^{n},
$$

and since it is plain that $S_{1}^{n} * T_{n}=n S_{1}^{n}$, so that $S_{1}^{n} *\left(T_{n}-n\right)=0$, the minimum polynomial of $T_{n}$ is

$$
P_{n}(x)=(x-n) \prod_{k=0}^{n-2}(x-k) .
$$

This shows that $T_{n}$ is semisimple, and allows an easy construction of the spectral projectors. 
Let us start with the kernel. The projector is given by $f \mapsto f * D_{n}$ where

$$
D_{n}=\frac{\left(T_{n}-1\right) *\left(T_{n}-2\right) * \cdots *\left(T_{n}-n+2\right) *\left(T_{n}-n\right)}{(-1)(-2) \cdots(2-n)(-n)}
$$

but since $T_{n}-n+1$ is invertible, one can take as well

$$
D_{n}=\frac{(-1)^{n}}{n !}\left(T_{n}-1\right)_{* n}
$$

where $(x)_{n}=x(x-1) \cdots(x-n+1)$, and the star means evaluation with the internal product. This is a better choice, since we have now a simple generating series for all these projectors,

$$
\sum_{n \geq 0} D_{n} x^{n}=e^{-x S_{1}} \cdot \sigma_{x}=\sigma_{x}((1-\mathbb{E}) A) .
$$

Indeed, we have $(x-1)_{n}=(x)_{n}-n(x-1)_{n-1}$, so that

$$
\frac{(-1)^{n}}{n !}(T-1)_{* n}=\frac{\left(-S_{1}\right)^{n}}{n !} \sigma_{1}-\frac{(-1)^{n-1}}{(n-1) !}(T-1)_{* n-1}=\sum_{k=0}^{n} \frac{\left(-S_{1}\right)^{k}}{k !} \sigma_{1} \text {. }
$$

The same reasoning shows that the projectors $D_{n, k}$ on the eigenspaces of $k$ are given by the generating series

$$
D(t, u)=\sum_{n \geq 0} \sum_{k=0}^{n} t^{n-k} u^{k} D_{n, k}=e^{(u-t) S_{1}} \sigma_{1},
$$

which is $\sigma_{1}(t A-(t-u) \mathbb{E} A)$, so that these elements coincide with those defined by (78).

\subsection{Characters of the associated modules}

The Frobenius characteristic of the left ideal of $\mathbb{C S}_{n}$ generated by the idempotents $\delta_{n, k}$ corresponding to $D_{n, k}$ via the identification $\sigma \leftrightarrow \mathbf{F}_{\sigma}$ can now be calculated as follows (compare [32, Corollary 4.2]).

Since $\delta_{n, k}$ is an idempotent, its characteristic $\operatorname{ch}\left(\delta_{n, k}\right)$ coincides with its cycle in$\operatorname{dex} Z\left(\delta_{n, k}\right)$. By the Gessel-Reutenauer formula [17], the coefficient of $p_{\mu}$ in $Z\left(\delta_{n, k}\right)$ is equal to $\left\langle D_{n, k}, L_{\mu}\right\rangle$, where $\underline{F}$ means the commutative image of the noncommutative symmetric function $F$, and for $v=1^{n_{1}} 2^{n_{2}} \ldots$,

$$
L_{v}=h_{n_{1}}\left[\ell_{1}\right] h_{n_{2}}\left[\ell_{2}\right] \cdots, \quad \ell_{n}=\frac{1}{n} \sum_{d \mid n} \mu(d) p_{n / d}^{d}
$$

$\mu$ denoting here the Moebius function. Hence, the generating function of all the cycle indexes is

$$
Z_{Y}(t, u)=\langle D(t, u ; X), L(X, Y)\rangle_{X}=\langle D(t, u ; X), L(Y, X)\rangle_{X}
$$


by the symmetry formula [34]

$$
L(X, Y)=L(Y, X)=\sum_{\mu} L_{\mu}(X) p_{\mu}(Y)=\prod_{n \geq 1} \sigma_{p_{n}(X)}\left[\ell_{n}(Y)\right] .
$$

Plugging this last expression into the scalar product and dualizing, we obtain

$$
\prod_{n \geq 1} \sigma_{p_{n}(t+(u-t) \mathbb{E})}\left[\ell_{n}(Y)\right]=\frac{\sigma_{1}((u-t) Y)}{1-t p_{1}(Y)} .
$$

In particular, specializing at $Y=\mathbb{E}$ gives that the dimension of $\mathbb{C S}_{n} \delta_{n, k}$ is $d_{n, k}$, the number of permutations in $\mathfrak{S}_{n}$ with exactly $k$ fixed points.

It is also easy to obtain the expansion of $Z_{Y}(t, u)$ as a combination of the $L_{\mu}(Y)$. Indeed, writing $D(t, u ; x)=\sigma_{1}((u-t) \mathbb{E} X+t X)$, we have $\left\langle D(t, u), p_{\mu}\right\rangle=$ $u^{m_{1}} \prod_{i \geq 2} t^{m_{i}}$, where $m_{i}$ is the multiplicity of the part $i$ in $\mu$. Hence,

$$
Z\left(\delta_{n, k}\right)=\sum_{m_{1}(\mu)=k} L_{\mu}
$$

We note that this is the quasi-symmetric generating function of the permutations with exactly $k$ fixed points. Note that $Z(D(t))$ is the commutative image of the generating series of desarrangements (23).

\section{$4.4 q$-derangement numbers}

From the above considerations, one can easily derive a (known) closed formula for the $q$-derangement numbers (compare [32, Theorem 4.5])

$$
d_{n}(q):=\sum_{\sigma \in D_{n}} q^{\mathrm{maj} \sigma}
$$

Indeed,

$$
\begin{aligned}
d_{n}(q) & =\left\langle\sum_{\sigma \in D_{n}} \mathbf{F}_{\sigma}, \sum_{\tau \in \mathfrak{S}_{n}} q^{\operatorname{maj}(\tau)} \mathbf{G}_{\tau}\right\rangle_{\mathbf{F Q S y m}} \\
& =\sum_{\sigma \in D_{n}|I|=n} \sum^{\operatorname{maj}(I)}\left\langle F_{C(\sigma)}, R_{I}\right\rangle=\left\langle\sum_{m_{1}(\mu)=0} L_{\mu}, K_{n}(q)\right\rangle .
\end{aligned}
$$

Hence,

$$
\begin{aligned}
\sum_{n \geq 0} d_{n}(q) \frac{x^{n}}{(q)_{n}} & =\left\langle\sigma_{1}\left[\sum_{n \geq 2} x^{n} l_{n}\right], \sigma_{1}\left(\frac{X}{1-q}\right)\right\rangle \\
& =\left\langle\frac{\lambda_{-x}}{1-x p_{1}}, \sigma_{1}\left(\frac{X}{1-q}\right)\right\rangle=\lambda_{-x}\left(\frac{1}{1-q}\right)\left(1-\frac{x}{1-q}\right)^{-1} \\
& =\frac{1-q}{1-x-q} \prod_{n \geq 0}\left(1-x q^{n}\right)
\end{aligned}
$$


so that finally [36]

$$
d_{n}(q)=[n] ! \sum_{k=0}^{n} \frac{(-1)^{k}}{[k] !} q^{\left(\begin{array}{c}
k \\
2
\end{array}\right)}
$$

4.5 Characters from Lie idempotents

The expression

$$
\operatorname{ch}\left(\delta_{n, k}\right)=\sum_{m_{1}(\lambda)=k} L_{\lambda}
$$

is also a consequence of the following type of expressions:

$$
D_{n, k}=\sum_{m_{1}(\lambda)=k} E_{\lambda}[\pi]
$$

in the notation of [19, Theorem 3.16], for some sequence $\pi=\left(\pi_{n}\right)$ of Lie idempotents in descent algebras. Indeed, [19, Theorem 3.21], implies then that the character is given by (140). We have already seen one such expression with $\pi_{n}=\zeta_{n}$, the Zassenhaus idempotents. We can also write one involving the Hausdorff series. Writing as usual

$$
\Phi=\sum_{n \geq 1} \phi_{n}=\log \sigma_{1}
$$

(the Solomon idempotents), we have

$$
\sum_{n \geq 0} D_{n}=e^{-\phi_{1}} e^{\Phi}=e^{H\left(-\phi_{1}, \Phi\right)}
$$

where $H$ is the Hausdorff series. Taking $\pi_{1}=\phi_{1}$ and $\pi_{n}=H_{n}\left(-\phi_{1}, \Phi\right)$ for $n \geq 2$, we obtain a sequence of Lie idempotents (see, e.g., [19], Theorem 3.1), from which it is easy to build a decomposition of the identity

$$
\sigma_{1}=e^{\pi_{1}} \exp \left\{\sum_{n \geq 2} \pi_{n}\right\}
$$

and more explicitly,

$$
S_{n}=\sum_{r+s=n} \frac{1}{r ! s !} \sum_{\ell(J)=r,|J|=n-s, 1 \notin J} \pi^{1^{s}, J} .
$$

This gives in particular the decomposition (141), with, for a partition $\lambda$ such that $m_{1}(\lambda)=0$,

$$
E_{\lambda}(\pi)=\frac{1}{\ell(\lambda) !} \sum_{I \downarrow \lambda} \pi^{I},
$$

where $I \downarrow \lambda$ means that the nondecreasing rearrangement of the composition $I$ is the partition $\lambda$. 


\subsection{Eigenbases of $t_{n}$}

From Proposition 7.4 of [11], we know the image a projector of the type (146). It is formed of weighted symmetrizations of Lie elements. With the above $\pi_{n}$, the distribution is uniform, so that the kernel consists in ordinary symmetrized products of Lie elements. Concretely, a basis of $\operatorname{Ker} t_{n}$ in $\mathbb{C S}_{n}$ is, for example,

$$
\left(\gamma_{1} \theta_{\lambda_{1}}, \gamma_{2} \theta_{\lambda_{2}}, \ldots, \gamma_{r} \theta_{\lambda_{r}}\right)
$$

where $(a, b, c)=a b c+a c b+b a c+b c a+c a b+c b a$, and so on (symmetrized products), the $\gamma_{k}$ are the minimal representatives of the cycles of a derangement, $\theta_{n}=[[\cdots[1,2], 3], \cdots n]$ is a Dynkin element, and $\lambda$ runs over partitions without part 1 .

For example, a basis of $\operatorname{Ker} t_{4}$ of dimension $d_{4}=9$ is given by the elements $[[[1, a], b], c]$ with $a b c$ running over permutations of 234 , for $L_{4}$, and by the three symmetrized products ([1, 2], [3, 4]), ([1, 3], [2, 4]), and ([1, 4], [2, 3]) for $L_{22}$.

Bases of the other eigenspaces are obtained by the same process, using weighted symmetrizations as indicated in [11]. Indeed, (145) shows that a basis of the eigenspace with eigenvalue $s$ is given by

$$
\left(\gamma_{1} \theta_{\lambda_{1}}, \gamma_{2} \theta_{\lambda_{2}}, \ldots, \gamma_{r} \theta_{\lambda_{r}}\right) \cdot\left(j_{1} \amalg j_{2} \amalg \cdots \amalg j_{s}\right)
$$

where $\gamma_{k}$ are the minimal representatives of the cycles of length at least 2 a permutation of cycle type $\left(\lambda, 1^{s}\right)$ having $s$ fixed points $j_{1}, j_{2}, \ldots, j_{s}$.

To continue with $n=4$, a basis of the 1 eigenspace is $[[i, j], k] \cdot l(i<j, k, i j k l$ a permutation of 1234), dimension 8 , and a basis of the 2-eigenspace is given by

$$
[i, j] \cdot(k l+l k), \quad i<j, k<l
$$

where $i j k l$ is a permutation of 1234 (dimension 6). Finally, the 4-eigenspace is one dimensional and generated by the full symmetrizer.

Using the $\zeta_{n}$ instead of the $\pi_{n}$, we can replace symmetrized products by ordinary products of homogeneous Lie polynomials taken in nondecreasing order of the degrees.

The idempotents $\delta_{n, k}$ have been first studied by M. Schocker [32] (apparently unaware of previous works on the subject and of their relation with the Tsetlin library).

\subsection{A basis of FQSym ${ }^{\sharp}$}

We have seen that the $(1-\mathbb{E})$-transform is a bialgebra morphism in FQSym. Hence, its image $\mathbf{F Q S y m}^{\sharp}$ is a Hopf subalgebra. The $\mathfrak{S}_{n}$-module $\Delta_{n, k}$ generated by $\delta_{n, k}$ can be identified with

$$
\mathbf{F Q S y m}_{n}^{\sharp}=\mathbf{F Q S y m}_{n} * D_{n, k},
$$

so that

$$
\operatorname{dim} \mathbf{F Q S y m}{ }_{n}^{\sharp}=d_{n} \text {. }
$$


It is therefore desirable to find a basis of FQSym ${ }^{\sharp}$ labeled by derangements, or some other set of permutations naturally in bijection with these. As we shall see, the natural transformation involved here is simply a version of Foata's first fundamental transformation [14].

Let $\gamma_{n}$ be the cycle

$$
\gamma_{n}:=n 12 \ldots n-1
$$

so that

$$
\mathbf{S}^{\gamma_{n}}=T_{n}=S_{1} S_{n-1}=R_{n}+R_{1, n-1}=\sum_{\sigma \in 1 \amalg 23 \ldots n} \mathbf{F}_{\sigma} .
$$

Since the $\sharp$-transform is a morphism for the product of FQSym,

$$
\mathbf{S}^{\gamma_{n}} * \sigma_{1}^{\sharp}=S_{1}^{\sharp} S_{n-1}^{\sharp}=0,
$$

and, for any permutation $\sigma \in \mathfrak{S}_{n}$,

$$
\left(\mathbf{F}_{\sigma} * \mathbf{S}^{\gamma_{n}}\right)^{\sharp}=\mathbf{F}_{\sigma} * \mathbf{S}^{\gamma_{n}} * \sigma^{\sharp}=0 .
$$

Recall that $i$ is a left-right minimum of $\sigma$ if

$$
\sigma_{j}>\sigma_{i} \text { for all } j<i \text {. }
$$

Let $X_{n}$ be the set of permutations of $\mathfrak{S}_{n}$ such that $\sigma \cdot 0$ does not have two consecutive left-right minima (that is, $\sigma$ does not end by 1 and does not have two consecutive LR-minima), and let $Y_{n}=\mathfrak{S}_{n} \backslash X_{n}$.

Lemma 4.1 For $\sigma \in Y_{n}$, write

$$
\sigma \cdot 0=\cdots \sigma_{i} \sigma_{i+1} \cdots
$$

where $i$ and $i+1$ is the first pair of consecutive LR-minima, and let

$$
\sigma^{\prime}=\sigma_{i} \cdot \sigma_{1} \cdots \hat{\sigma}_{i} \cdots \sigma_{n}
$$

be the permutation obtained by moving $\sigma_{i}$ at the first position, leaving the remaining letters unchanged, and removing the zero in the end. Then

$$
\mathbf{F}_{\sigma^{\prime}} * \mathbf{S}^{\gamma_{n}}=\mathbf{F}_{\sigma}+\sum_{\tau \in T} \mathbf{F}_{\tau}
$$

where the permutations of $T$ are lexicographically smaller than $\sigma$.

Proof The expression

$$
\mathbf{F}_{\sigma^{\prime}} * \mathbf{S}^{\gamma_{n}}=\sum_{\tau \in \sigma_{i} \uplus \sigma_{1} \ldots \hat{\sigma}_{i} \ldots \sigma_{n}} \mathbf{F}_{\tau}
$$

contains $\mathbf{F}_{\sigma}$ and, since $\sigma_{i}$ is a LR-minimum, $\sigma$ is the maximal element of the previous sum. 
For a permutation $\sigma$ with LR-minima $i_{1}, \ldots, i_{p}$, let

$$
\phi(\sigma)=\left(\sigma_{1} \ldots \sigma_{i_{1}-1}\right)\left(\sigma_{i_{1}} \ldots \sigma_{i_{2}-1}\right) \ldots\left(\sigma_{i_{p}} \ldots \sigma_{n}\right) \text {, }
$$

where each parenthesis represents a cycle. For example, with $\sigma=62781453$,

$$
\phi(\sigma)=(6)(278)(1453)=47153682 .
$$

This is Foata's first fundamental transformation (up to reversing the order on the integers), hence a bijection. Clearly, $\phi(\sigma)$ has fixed points whenever $\sigma \in Y_{n}$, so $\phi$ induces a bijection between $X_{n}$ and derangements of $\mathfrak{S}_{n}$.

From Lemma 4.1, we see that the elements

$$
\left(\mathbf{F}_{\sigma}^{\sharp}\right)_{\sigma \in X_{n}}
$$

$\operatorname{span} \mathbf{F Q S y m}_{n}^{\sharp}$. Since $\left|X_{n}\right|=d_{n}=\operatorname{dim} \mathbf{F Q S y m} \mathbf{m}_{n}^{\sharp}$, we have finally

Theorem 4.2 The $\mathbf{F}_{\sigma}^{\sharp}$ for $\sigma \in X_{n}$ form a basis of $\mathbf{F Q S y m}^{\sharp}$.

The sets $X_{n}$ have an interesting structure.

Theorem 4.3 The set $X_{n}$ is an ideal of the left weak order on $\mathfrak{S}_{n}$. Its maximal elements are the left-shifted concatenations

$$
w_{I}:=w_{i_{1}} \triangleright \cdots w_{i_{r}},
$$

where $w_{i}:=1$ i $i-1, \ldots, 2$, composition I has no part 1 , and $\alpha \triangleright \beta=\alpha[\ell] \cdot \beta$ if $\beta \in \mathfrak{S}_{\ell}$.

Proof To show that $X_{n}$ is an ideal, we will prove that if $s_{i}$ denotes the elementary transposition $(i, i+1)$, then $\sigma \in Y_{n}$ and $\operatorname{inv}\left(s_{i} \sigma\right)=\operatorname{inv}(\sigma)+1$, implies $s_{i} \sigma \in Y_{n}$.

If $\sigma_{k}=r>s=\sigma_{k+1}$ are consecutive LR-minima of $\sigma$, they will remain so for $s_{i} \sigma$, unless $i=r-1, r, s-1$, or $s$. Since $s_{i} \sigma$ has one inversion more than $\sigma$, we can exclude the case $i=r-1: r$ being a LR-minimum, $r-1$ cannot be to the left of $r$ in $\sigma$. We can also exclude $i=s-1$ for the same reason. If $i=r$, then $r$ is exchanged with $r+1$, which has to be to its right in $\sigma$, so that again $\sigma_{k}$ and $\sigma_{k+1}$ are consecutive LR-minima in $s_{i} \sigma$. The same reasoning applies with $i=s$. Hence, $Y_{n}$ is a coideal, and consequently $X_{n}$ is an ideal.

Now, the elements $w_{I}$ are clearly in $X_{n}$ when $I$ has no part 1 , and any exchange of consecutive values creating an inversion in such a $w_{I}$ would create a pair of consecutive LR-minima. So these $w_{I}$ are maximal elements of the ideal $X_{n}$.

Conversely, consider $\sigma \in X_{n}$ maximal. Then consider the suffix $s$ of $\sigma$ beginning with 1 . The maximality condition of $\sigma$ implies that if $t$ belongs to that suffix, then $t-1$ also belongs to it. So this prefix is a permutation of an $\mathfrak{S}_{|s|}$, then should be $1|s| \ldots 2$. The same now works by induction on the permutation $\tau$ defined by $\sigma=$ $\tau \gg s$. 
For example, with $n=5$, we get the following three maximal elements of $X_{n}$ :

$15432,35412,45132$.

The same proof can be adapted to the case of permutations that are images by $\phi$ of permutations with at most $k$ fixed points. Let $X_{n}^{(k)}$ be the image by $\phi$ of permutations with at most $k$ fixed points. Then $X_{n}^{(k)}$ is the set of permutations with at most $k$ consecutive LR-minima.

Theorem 4.4 The set $X_{n}^{(k)}$ is a ideal of the left weak order on $\mathfrak{S}_{n}$. Its maximal elements are the $w_{I}$ where I runs over compositions with

- either $k-1$ ones and the remaining parts equal to 2 ,

- or exactly $k$ ones.

Proof The fact that $X_{n}^{(k)}$ is an ideal comes from the same idea as before: all permutations greater than a given permutation $\sigma$ for the left weak order have LR-minima at the same position.

By the same argument as in the previous theorem, the maximal elements must be some $w_{I}$, where $I$ has at most $k$ ones. Now, it is clear that

$$
w_{I}<w_{J}
$$

in the left weak order iff $I$ can be obtained from $J$ by gluing parts equal to 1 with their next part. So the compositions described in the statement are definitely maximal elements. And since all compositions with at most $k$ ones can be obtained from these ones by the gluing process, this ends the proof.

Here is a table (Table 2) of the number of maximal elements of $X_{n}^{(k)}$. Note that the first column is obviously given by Fibonacci numbers since these indeed count the number of compositions of $n$ in parts at least 2 . The other columns are not known to [35] and neither is the sequence of row sums.

Table 2

\begin{tabular}{lrrrrrrrrr}
\hline$n \backslash k$ & 0 & 1 & 2 & 3 & 4 & 5 & 6 & 7 & 8 \\
\hline 1 & 0 & 1 & & & & & & \\
2 & 1 & 1 & 1 & & & & & \\
3 & 1 & 2 & 2 & 1 & & & & \\
4 & 2 & 3 & 3 & 3 & 1 & & & & \\
5 & 3 & 5 & 6 & 4 & 4 & 1 & & & \\
6 & 5 & 9 & 9 & 10 & 5 & 5 & 1 & & \\
7 & 8 & 15 & 19 & 14 & 15 & 6 & 6 & 1 & \\
8 & 13 & 27 & 31 & 34 & 20 & 21 & 7 & 7 & 1 \\
\hline
\end{tabular}


But there exists a simple formula giving the number of maximal elements, coming directly from their characterization:

$$
m_{n, k}:=\left(\begin{array}{c}
(n+k-1) / 2 \\
k-1
\end{array}\right)+\sum_{\ell=0}^{\left\lfloor\frac{n-k}{2}\right\rfloor}\left(\begin{array}{c}
\ell+k \\
k
\end{array}\right)\left(\begin{array}{c}
n-k-\ell-1 \\
\ell-1
\end{array}\right)
$$

with the convention that a binomial coefficient with entries not in the natural numbers is zero.

\subsection{Other bases of FQSym ${ }^{\sharp}$}

Conjecture 4.5 Let $<$ be the order on permutations defined by

$$
\sigma<^{\prime} \tau \Longleftrightarrow \phi(\sigma)<\operatorname{lex} \phi(\tau) \text {. }
$$

Then the matrix of $\mathbf{S}^{\sigma \sharp}$ of the $\mathbf{S}$ basis is triangular. Moreover, the diagonal values are 1 for the elements of $X_{n}$ and 0 for $Y_{n}$.

For example, here are the matrices for $n=2,3$, and 4 (Fig. 1) where the zero entries have been represented by dots to enhance readability.

The permutations are ordered as follows:

$$
\begin{aligned}
& \text { [21, 12], [321, 312, 231, 123, 132, 213]. } \\
& \text { [4321, 4312, 4231, 4123, 4132, 4213, 3421, 3412, } \\
& \text { 2341, 1234, 1243, 2314, 2431, 1423, 3241, 2134, } \\
& \text { 3142, 1324, 1432, 2413, 2143, 3214, 1342, 3124]. } \\
& \left(\begin{array}{cc}
. & -1 / 2 \\
. & 1
\end{array}\right) \quad\left(\begin{array}{cccccc}
\cdot & . & . & 1 / 3 & -1 / 3 & 2 / 3 \\
. & . & . & -1 & . & -1 \\
. & . & . & . & . & . \\
. & . & . & 1 & . & 1 \\
. & . & . & . & 1 & -1 \\
. & . & . & . & . & .
\end{array}\right) .
\end{aligned}
$$

\section{Other combinatorial Hopf algebras}

\subsection{The algebras PQSym and CQSym}

There is an internal product on PQSym extending that of WQSym* [26]. The \#transform is defined in PQSym (it contains Sym as a subalgebra), but PQSym * WQSym$^{*} \subseteq$ WQSym $^{*}$, so that $\mathbf{P Q S y m} \mathbf{m}^{\sharp}=\mathcal{W}^{\sharp}$, and we get nothing new.

Similarly, for the Catalan algebra CQSym [28], we have

$$
\mathbf{C Q S y m} * \mathbf{S y m} \subseteq \mathbf{S y m},
$$




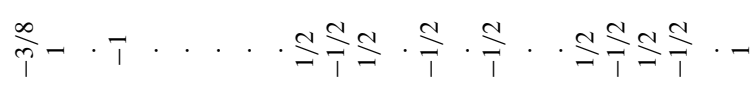

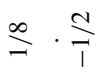

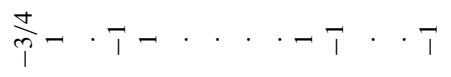

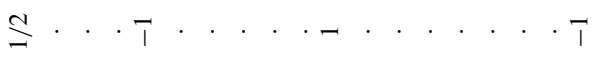

$\frac{m}{2} \stackrel{1}{=}$

$\stackrel{+}{i}$

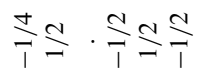

$\frac{m}{m} \cdot \cdot \cdot \cdot \cdot \cdot \frac{1}{=} \cdot \frac{1}{N} \cdot-$

$\underset{\mathrm{I}}{\mathrm{I}}-\mathrm{T} \cdot \cdot \cdot \cdot \cdot \cdot \cdot \cdot \cdot \cdot$

$\stackrel{-1}{\stackrel{1}{N}}$

$\stackrel{\infty}{m} \stackrel{\sim}{\imath} \cdot \uparrow-\cdot \cdot \cdot-\urcorner$

$\stackrel{ \pm}{ \pm} \cdot \cdot \cdot$

$\stackrel{\infty}{\longrightarrow} \stackrel{\imath}{=}$

$\stackrel{+}{-} \stackrel{1}{=}$

$\cdot \frac{1}{2}-$ 
so that

$$
\mathrm{CQSym}^{\sharp}=\mathrm{Sym}^{\sharp} \text {. }
$$

\subsection{The algebra of planar binary trees PBT}

The Loday-Ronco algebra of planar binary trees is not stable by the $\sharp$-transform. Since PBT is the subalgebra of FQSym generated by the $S^{\sigma}$ where $\sigma$ avoids the pattern 132 (see [13]), we have, for example,

$$
S^{213^{\sharp}}=S^{123}-S^{132}-S^{312}+\frac{2}{3} S^{321} \notin \mathbf{P B T} .
$$

\section{However, PBT $\mathbf{T}^{\sharp}$ is a well-defined Hopf subalgebra of FQSym.}

Conjecture 5.1 The algebra $\mathbf{P B} \mathbf{T}^{\sharp}$ is free over the set $\mathbf{P}_{T}^{\sharp}$, where $T$ runs over trees with at least two nodes, and such that the right subtree of the root is empty.

In particular, the conjecture implies that the dimension of the homogeneous components $\mathbf{P B T}_{n}^{\sharp}$ are given by the Fine numbers [8, 35], sequence A000957:

$$
1,01261857186,622, \ldots
$$

\section{References}

1. Aguiar, M., Novelli, J.-C., Thibon, J.-Y.: Unital versions of the higher order peak algebras, arXiv:0810.4634, FPSAC'09 (Linz)

2. Bidigare, P., Hanlon, P., Rockmore, D.: A combinatorial description of the spectrum of the Tsetlin library and its generalization to hyperplane arrangements. Duke Math. J. 99, 135-174 (1999)

3. Bergeron, N., Hivert, F., Thibon, J.-Y.: The peak algebra and the Hecke-Clifford algebras at $q=0$. J. Comb. Theory A 117, 1-19 (2004)

4. Blessenohl, D., Laue, H.: The module structure of Solomon's descent algebra. J. Aust. Math. Soc. 72(3), 317-333 (2002)

5. Désarménien, J.: Une autre interprétation du nombre de dérangements. Sémin. Lothar. Comb. 8, 11$16(1984)$

6. Désarménien, J., Wachs, M.: Descentes sur les dérangements et mots circulaires. Sémin. Lothar. Comb. 19, 13-21 (1988)

7. Désarménien, J., Wachs, M.: Descent classes of permutations with a given number of fixed points. J. Comb. Theory Ser. A 64, 311-328 (1993)

8. Deutsch, E., Shapiro, L.: A survey of the Fine numbers. Discrete Math. 241, 241-265 (2001)

9. Duchamp, G., Hivert, F., Thibon, J.-Y.: Noncommutative symmetric functions VI: free quasisymmetric functions and related algebras. Int. J. Algebra Comput. 12, 671-717 (2002)

10. Duchamp, G., Hivert, F., Novelli, J.-C., Thibon, J.-Y.: Noncommutative symmetric functions VII: free quasi-symmetric functions revisited, arXiv:0809.4479

11. Duchamp, G., Klyachko, A., Krob, D., Thibon, J.-Y.: Noncommutative symmetric functions III: Deformations of Cauchy and convolution algebras. Discrete Math. Theor. Comput. Sci. 1, 159-216 (1997)

12. Gelfand, I.M., Krob, D., Lascoux, A., Leclerc, B., Retakh, V.S., Thibon, J.-Y.: Noncommutative symmetric functions. Adv. Math. 112, 218-348 (1995)

13. Hivert, F., Novelli, J.-C., Thibon, J.-Y.: The algebra of binary search trees. Theor. Comput. Sci. 339, 129-165 (2005)

14. Lothaire, N.: Combinatorics on Words. Cambridge University Press, Cambridge (1997) 
15. Garsia, A.M., Reutenauer, C.: A decomposition of Solomon's descent algebra. Adv. Math. 77, 189262 (1989)

16. Garsia, A.M., Wallach, N.: $r$-QSym is free over Sym. J. Comb. Theory A 114, 704-732 (2007)

17. Gessel, I., Reutenauer, C.: Counting permutations with given cycle structure and descent set. J. Comb. Theory A 64, 189-215 (1993)

18. Hivert, F.: Combinatoire des fonctions quasi-symétriques. Thèse de Doctorat, Marne-La-Vallée (1999)

19. Krob, D., Leclerc, B., Thibon, J.-Y.: Noncommutative symmetric functions II: Transformations of alphabets. Int. J. Algebra Comput. 7, 181-264 (1997)

20. Lascoux, A.: Symmetric Functions and Combinatorial Operators on Polynomials. CBMS Regional Conference Series in Mathematics, vol. 99. American Math. Soc., Providence (2003), xii+268 pp.

21. Lascoux, A., Schützenberger, M.P.: Formulaire Raisonné de Fonctions Symétriques. Publ. Math. Univ. Paris 7, Paris (1985)

22. Macdonald, I.G.: Symmetric Functions and Hall Polynomials, 2nd edn. Oxford University Press, London (1995)

23. Malvenuto, C., Reutenauer, C.: Duality between quasi-symmetric functions and the Solomon descent algebra. J. Algebra 177, 967-982 (1995)

24. Novelli, J.-C., Saliola, F., Thibon, J.-Y.: Representation theory of the higher order peak algebras. Preprint 0906.5236 [math.CO]

25. Novelli, J.-C., Thibon, J.-Y.: A Hopf algebra of parking functions. In: FPSAC'04, Vancouver (2004)

26. Novelli, J.-C., Thibon, J.-Y.: Parking functions and descent algebras. Ann. Comb. 11, 59-68 (2007)

27. Novelli, J.-C., Thibon, J.-Y.: Polynomial realizations of some trialgebras. In: FPSAC'06. Also preprint arXiv:math.CO/0605061

28. Novelli, J.-C., Thibon, J.-Y.: Hopf algebras and dendriform structures arising from parking functions. Fundam. Math. 193, 189-241 (2007)

29. Novelli, J.-C., Thibon, J.-Y.: Noncommutative symmetric functions and Lagrange inversion. Adv. Appl. Math. 40, 8-35 (2008)

30. Patras, F., Schocker, M.: Twisted Descent Algebras and the Solomon-Tits Algebra. Adv. Math. 199, 151-184 (2006)

31. Saliola, F.: On the quiver of the descent algebra. J. Algebra 320, 3866-3894 (2008)

32. Schocker, M.: Idempotents for derangement numbers. Discrete Math. 269, 239-248 (2003)

33. Schocker, M.: The module structure of the Solomon-Tits algebra of the symmetric group. J. Algebra 301(2), 554-586 (2006)

34. Scharf, T., Thibon, J.-Y.: A Hopf algebra approach to inner plethysm. Adv. Math. 104, 30-58 (1994)

35. Sloane, N.J.A.: The On-Line Encyclopedia of Integer Sequences (electronic), http://www.research.att. com/ njas/sequences/

36. Wachs, M.: On $q$-derangement numbers. Proc. Am. Math. Soc. 106, 273-278 (1989) 